\title{
13. MICROBIAL ACTIVITY IN THE ALTERATION OF GLASS FROM PILLOW LAVAS FROM HOLE 896A ${ }^{1}$
}

\author{
Harald Furnes, ${ }^{2}$ Ingunn H. Thorseth, ${ }^{2}$ Ole Tumyr, ${ }^{2}$ Terje Torsvik,${ }^{3}$ and Martin R. Fisk ${ }^{4}$
}

\begin{abstract}
Within the altered parts of the glass rim of pillow lavas of Hole $896 \mathrm{~A}$, at depths at least $432 \mathrm{~m}$ below seafloor ( $237 \mathrm{~m}$ below the top of volcanic basement), microbes have been identified. This is indicated by the size and shapes of alteration textures and verified by the presence of DNA and extreme accumulation of $\mathrm{K}_{2} \mathrm{O}$. This demonstrates the existence of a volcanic subterranean biosphere. The microbially processed parts of the glass show wide scatter with respect to all major elements, which may be attributed to active cells. Compared to the host basaltic glass, $\mathrm{CaO}$ and $\mathrm{Na}_{2} \mathrm{O}$ are invariably depleted, as are $\mathrm{SiO}$. and $\mathrm{MgO}_{\text {gen- }}$ erally. $\mathrm{Al}_{2} \mathrm{O}_{3}, \mathrm{FeO}(\mathrm{t})$, and $\mathrm{TiO}_{2}$ show either depletion or enrichment, $\mathrm{K}_{2} \mathrm{O}$ is invariably enriched, and $\mathrm{P}_{2} \mathrm{O}_{5}$ may be enriched. Microbes living on, and causing dissolution of, basaltic glass may accommodate elements released from it within the cells, and thus function as individual element reservoirs. Microbes may also produce precipitates or water-soluble compounds. Hence, the microbial alteration of basaltic glass, which comprises a substantial volume of the volcanic component of the oceanic crust and an enormous surface area, may have a significant bearing on the mechanism for chemical exchange between oceanic crust and ocean water.
\end{abstract}

\section{INTRODUCTION}

Weathering, diagenesis, and hydrothermal alteration of silicates play a significant role in the chemical composition of natural waters and the global cycling of elements (Hiebert and Bennett, 1992). Basaltic glass is reactive and decomposes more readily than the associated minerals. Hence, in many natural deposits such as tephra and pillow basalts the main incipient element fluxes result from interaction between the glass phase and the aqueous solution. The alteration of basaltic glass, which comprises a significant component of the upper part of the oceanic crust, is thus considered a most important process in element exchange between seawater and the oceanic crust (Staudigel and Hart, 1983). Thus, the understanding of the factors that control alteration rate is of crucial importance.

It is well known that during low-temperature seawater alteration, basaltic glass alters to a brownish composite and geochemically heterogeneous material, commonly referred to as palagonite (e.g., Peacock, 1926; Moore, 1966; Hay and Iijima, 1968a, 1968b; Stokes, 1971; Honnorez, 1972; Jakobsson, 1972; Noack, 1979; Furnes and El-Anbaawy, 1980; Thorseth et al., 1991). Until recently, the alteration of glass has been viewed in terms of physical/chemical processes only, and different studies have led to contradictory models, such as (1) total (congruent) dissolution of glass followed by the successive precipitation of stable, hydrous substances and hence the formation of palagonite (e.g., Crovisier et al., 1987) and (2) selective (incongruent) leaching of cations replaced by $\mathrm{H}_{3} \mathrm{O}^{+}$and slower, partial dissolution of network-forming elements, which results in a $\mathrm{Si}$ rich, porous material, in which precipitation will accumulate elements (e.g., Berger et al., 1987). Chemical studies of alteration products from meteoric as well as submarine environments show

Alt, J.C., Kinoshita, H., Stokking, L.B., and Michael, P.J. (Eds.), 1996. Proc. ODP, Sci. Results, 148: College Station, TX (Ocean Drilling Program).

${ }^{2}$ Geological Institute, University of Bergen, Allegt. 41, 5007 Bergen, Norway. Furnes: Harald.Furnes@geol.uib.no; Thorseth: Ingunn.Thorseth@geol.uib.no; Tumyr: Ole.Tumyr@geol.uib.no

Department of Microbiology, University of Bergen, 5007 Bergen, Norway. Terje.Torsvik@im.uib.no

${ }^{4}$ College of Oceanographic and Atmospheric Sciences, Oregon State University, Corvallis, OR 97331-5503.U.S.A. Martin-Fisk@oce.orst.edu redistribution of most elements relative to the glass parent, but the changes may vary in magnitude and direction even on a micro scale, indicating the development of various microenvironments during alteration (Thorseth et al., 1991, 1992a, 1992b).

Textural studies using a scanning electron microscope (SEM) on subglacial hyaloclastites in Iceland and the 30 -yr-old shallow-marine to subaerial tephra on Surtsey (Iceland) (Jakobsson, 1978) have shown that bacteria and alteration are associated. Bacteria were identified (1) at the boundary between fresh glass and palagonite, (2) in etch-generated channels penetrating a few microns into the glass, and (3) in the porous, sponge-textured palagonite, with pores of the same size and shape as the bacteria (Thorseth et al., 1992b). From these observations, as well as previous studies on the biogenic effect on volcanic glass (Jones and Goodbody, 1982; Ross and Fisher, 1986), it was suggested that microbiota may play a significant role in the decomposition of basaltic glass, which has also been experimentally demonstrated (Thorseth et al., in press).

In this chapter, we report the occurrence of microbially produced textures along thin joints in the glassy margin of pillows at different levels in Ocean Drilling Program (ODP) Hole 896A. We further compare the geochemistry of the microbially processed parts with the adjacent fresh basaltic glass, and, finally, discuss the importance of microbial activity on basaltic glass in the ocean crust in terms of consequences and dimensions.

\section{ANALYTICAL METHOD Scanning Electron Microscopy}

For the SEM observations, a JEOL scanning microscope (JSM6400 ), connected to a TRACOR NORTHERN (TN 5600 Series II) energy-dispersive spectrometer system with a Z-MAX 30 (diamond window) detector, was used. The analyses were performed at an accelerating voltage of $20 \mathrm{kV}$. Thin sections selected for investigation were covered with an approximately 300 - $\AA$-thick film of carbon.

\section{Light and Epifluorescence Microscopy}

Thin sections, embedded in epoxy, were stained with $10 \mathrm{~g} / \mathrm{mL}$ DAPI (4,6 diamino-phenyl-indole, a fluorescent dye that specifically 
stains DNA) for $1 \mathrm{~min}$. Excess stain was removed by washing with distilled water. The method follows Porter and Feig (1980). Samples were examined in a Nikon Microphot microscope using light transmission and epifluorescence with an excitation light wavelength of $365 \mathrm{~nm}$ and an emission wavelength of $420 \mathrm{~nm}$.

\section{Electron Microprobe Analyses}

Most electron microprobe analyses were performed on a fully automated CAMECA CAMEBAX in Oslo, using an acceleration voltage of $15 \mathrm{kV}$ and a beam current of $5 \mathrm{nA}$. For calibration, appropriate mineral standards were used. Counting times were 10 and $5 \mathrm{~s}$ on the peak and background, respectively. Additional microprobe analyses were done with a four-spectrometer CAMECA SX-50 at Corvallis, using an accelerating voltage of $15 \mathrm{kV}$ and a beam current of $50 \mathrm{nA}$. Counting times were $10 \mathrm{~s}$, except for $\mathrm{Al}$ and $\mathrm{Ti}$, for which the counting times were $20 \mathrm{~s}$.

\section{DESCRIPTION OF INVESTIGATED MATERIAL}

Volcanic basement consisting of pillow basalts and minor massive flows and breccias was cored from 195.1 to $469 \mathrm{~m}$ below seafloor (mbsf) (Shipboard Scientific Party, 1993). The investigated material reported in this account comprises five samples from the rim of pillow lavas, of which the four deepest samples were the most extensively studied: Samples 148-896A-7R-1, 52-55 cm (255.75 mbsf]), 148-896A-9R-1, 17-21 cm (266.87 mbsf), 148-896A-11R-1, $73-75 \mathrm{~cm}$ (286.73 mbsf), 148-896A-11R-1, 111-113 cm (287.11 mbsf), and 148-896A-27R-1, 114-117 cm (431.64 mbsf) (for detailed petrography and geochemistry, see Shipboard Scientific Party, 1993). Above basaltic basement is a cover of $195 \mathrm{~m}$ of dominantly siliceous nannofossil oozes, described from the adjacent Hole 504B (Anderson et al., 1982).

\section{Textures}

In the 1- to 2-cm-thick glass rim of the pillows, there are cracks of different generations. Along most of the cracks are zones and irregular patches of altered glass (PI. 1). In many cases, the alteration patches are developed only on one side of a joint (e.g., Pl. 1, Figs. 1, 2). Also, a common appearance is sausage-like shapes along joints, as demonstrated in Plate 1, Figure 3. In all samples investigated there are clearly different generations of alteration patches along the joints, from those with no alteration, to the very incipient and advanced stages (PI. 1, Fig. 4). These patches consist mainly of aggregates of (1) small spherical bodies (type 1) $0.5-1 \mu \mathrm{m}$ in diameter, defining patches up to approximately $50 \mu \mathrm{m}$ thick on either side of the cracks; (2) two less dominant groups of larger spherical bodies 2-3 and 5-10 $\mu \mathrm{m}$ in diameter (types 2 and 3, respectively) (Pl. 2); and (3) vermicular bodies up to $70 \mu \mathrm{m}$ long (type 4) surrounded by fresh glass (PI. 3 ). These textures, by their size and shape, strongly indicate that they were produced by the etching of microbes, as reported from both naturally and experimentally altered samples (Thorseth et al., 1992a, 1992b, in press). Types 1 and 4 were found within all investigated depth levels, but types 2 and 3 were not found at the shallowest levels (in Samples 148-896A-7R-1, 52-55 cm, and 9R-1, 17-21 cm, at 255.75 and 266.87 mbsf, respectively).

A general feature in all samples investigated is that the smallest spherical alteration bodies, type 1, appeared first along joint surfaces. At a more advanced stage of alteration, the larger spherical alteration bodies (types 2 and 3), as well as the vermicular type 4, are apparent. Only at the alteration front, where interstitially, fresh glass is still present, is it possible to identify the four alteration types (PI. 2, Figs. 1,2 ; Pl. 3, Figs. 1-4). Where the glass has been totally altered, all textural evidence of microbial activity is generally obliterated.
The four different types of bodies were originally used for the convenience of description. To what extent the smallest spherical bodies (type 1 ) represent slices of bigger bodies (types 2 and 3 ), type 2 bodies represent slices of the bigger type 3 bodies, and types 2 and 3 represent sections perpendicular to tubes (type 4) is difficult to estimate. However, with respect to type 1 , it is common to find this size group only. Similarly, in some places, types 1 and 4 may be dominant, whereas in other places, types 2 and 3 may be well represented, without any traces of type 4 . Furthermore, there are no continuous size gradations between the spherical types 1,2 , and 3 . Therefore, we think that the four morphological and different-size types are real, and that they may represent areas produced by different types of microbes.

To verify the existence of microbes, independent of the textural evidence, the samples were investigated, prior and subsequent to DNA-specific DAPI staining, by ordinary light and epifluorescence microscopy. The result of this test is shown in Plate 4, Figures 1 and 2. The aggregates consisting of the smallest sized spherical bodies (type 1) fluoresce, in particular adjacent to the fresh glass, which demonstrates the presence of DNA-containing cells with a size similar to that of bacteria (Pl. 4, Fig. 2). Similar tests, with three different types of staining from that used for this account, were performed by Giovannoni et al. (this volume). These all gave the same result as reported here.

\section{GEOCHEMICAL RESULTS}

A total of 282 microprobe analyses were conducted for the present study. Forty-seven analyses of the fresh glass were performed (Table 1); of the microbially processed parts defining the different textures, $68,33,41$, and 55 analyses of types 1, 2, 3, and 4 were performed, respectively (Table 2 ). Thirty-eight analyses from four profiles across altered basaltic glass with a central joint (Table 3 ) were also made.

\section{Basaltic Glass}

The fresh glass analyses are shown in Table 1. Within analytical uncertainties, the chemistries of the upper four samples (148-896A7R-1, 52-55 cm; 9R-1, 17-21 cm; 11R-1, 73-75 cm; and 11R-1, 111-113 cm [255.75-287.11 mbsf]) are similar. The lowest sample (148-896A-27R-1, 114-117 cm [431.64 mbsf]), however, is slightly higher in $\mathrm{MgO}$ and lower in $\mathrm{TiO}_{2}$ and $\mathrm{Na}_{2} \mathrm{O}$ than the other four samples.

\section{Microbially Processed Glass}

The micropits and microchannels (Pls. 1-3), supposedly caused by microbial etching, contain amorphous material. Microprobe analyses of the various spherical (types 1-3) and vermicular (type 4) bodies are shown in Table 2. The totals of the analyses of the microbially processed glass show large variations, from 99.51 to $64.80 \mathrm{wt} \%$ (Table 2; Fig. 1). For the smallest spherical bodies (type 1), most of the totals range between 94 and 83 wt\%. A rather similar distribution is shown by the vermicular bodies (type 4), but the majority of the totals of these bodies range between 92 and $80 \mathrm{wt} \%$. Spherical types 2 and 3 define a rather similar distribution of the analytical totals and show a continuous range between 92 and $64 \mathrm{wt} \%$. The low, and highly different, totals may be caused by such factors as analytical problems, variable $\mathrm{H}_{2} \mathrm{O}$ content, and/or porosity, in addition to other components that were not analyzed by the electron microprobe (C, N, O, P, etc.). The range of analytical totals may to some extent reflect the interaction of beam and of secondary X-rays with the fresh glass. This may be suggested by the fact that type 1 , representing the smallest bodies, in general has the highest totals, which, in some cases, approach the composition of the fresh glass (Table 2). To avoid this problem as 
Table 1. Microprobe analyses of glass rims of pillow lavas, Hole 896A.

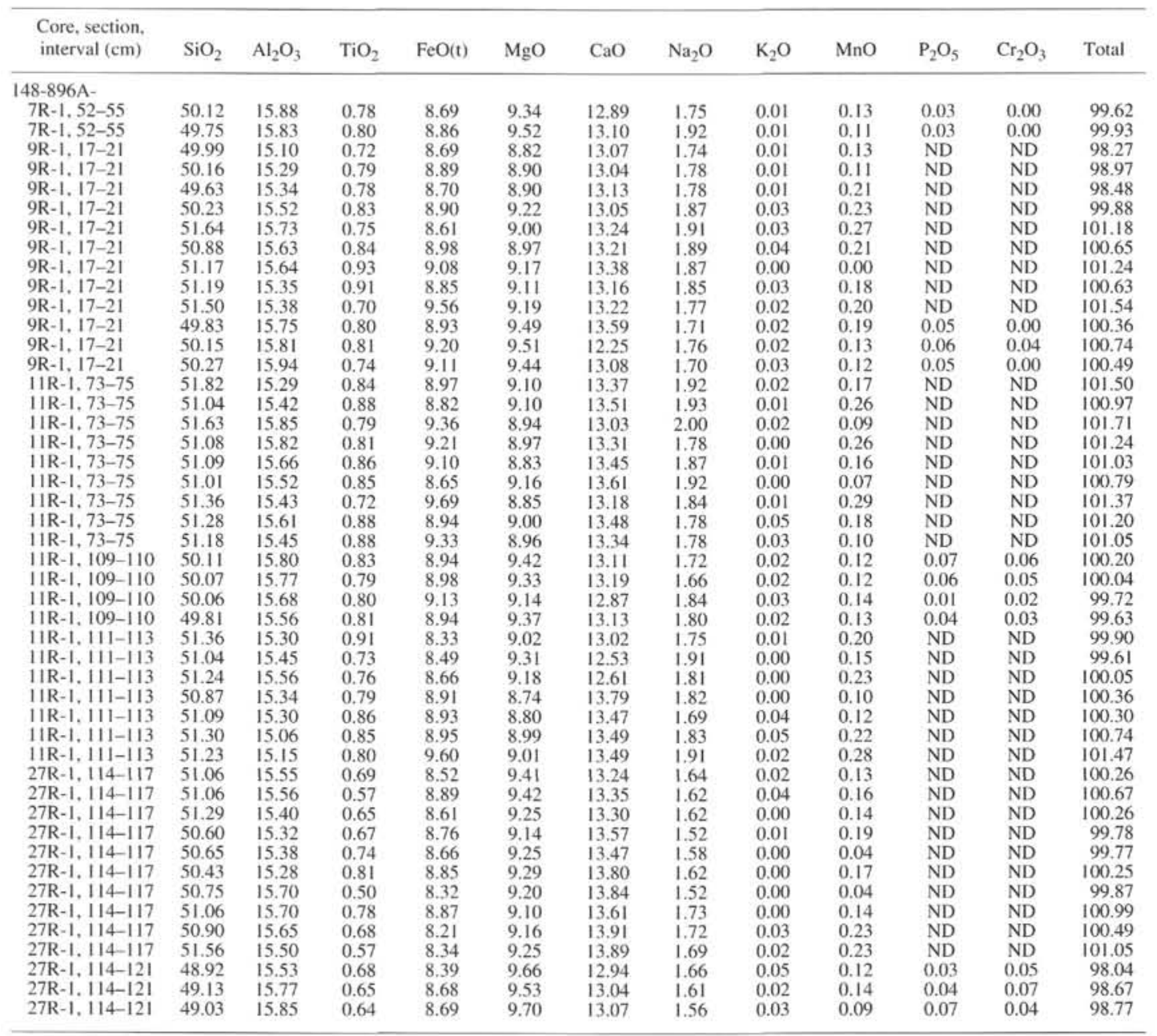

Notes: $\mathrm{FeO}(\mathrm{t})=$ total iron reported as $\mathrm{FeO} . \mathrm{ND}=$ not determined.

much as possible, we carefully selected the areas for analyses where clusters of several bodies occur. In a chemical study of palagonite by Thorseth et al. (1991), a good negative correlation between total wt\% oxides (approximately $90-50$ ) and percentage measured porosity (approximately $0-45$ ) could be demonstrated. The same phenomenon (i.e., the lower the total, the higher the porosity) may be the case in this study. However, we have not been able to check this possibility. In the following diagrams, the unmodified data are used.

Figures 2 through 5 are chemical variation diagrams of the morphologically different forms (types 1-4) at various depths. All four types show large variations with respect to the analyzed elements and, in general, the same pattern of scatter. Compared to the fresh basaltic glass, $\mathrm{CaO}$ and $\mathrm{Na}_{2} \mathrm{O}$ are invariably depleted, and $\mathrm{SiO}_{2}$ and $\mathrm{MgO}$ are commonly depleted. $\mathrm{Al}_{2} \mathrm{O}_{3}, \mathrm{FeO}(\mathrm{t}), \mathrm{TiO}_{2}$, and $\mathrm{P}_{2} \mathrm{O}_{5}$ show both depletion and enrichment, and $\mathrm{K}_{2} \mathrm{O}$ is strongly enriched. In general, $\mathrm{Na}_{2} \mathrm{O}$ shows progressive loss with increasing $\mathrm{K}_{2} \mathrm{O}$. Two elements that, to a large extent, show only minor deviation from the fresh basaltic glass are $\mathrm{FeO}(\mathrm{t})$ and $\mathrm{TiO}_{2}$; however, with the exception of type 2 (Fig. 3), there is no correlation between the two elements. With respect to types $1-3$, some of the analyses show an extreme depletion in $\mathrm{FeO}(\mathrm{t})$. The smallest bodies (type 1), which represent the dominant type, have a rather similar chemical composition (with respect to $\mathrm{Al}_{2} \mathrm{O}_{3}, \mathrm{FeO}(\mathrm{t})$, and $\mathrm{SiO}_{2}$ ) to fresh basaltic glass. The other three types are much more depleted in $\mathrm{SiO}_{2}$.
The morphological types may show pronounced differences in chemical composition related to the depth at which they occur (Fig. $6)$. This is particularly well demonstrated for the deepest sample (148-896A-27R-1, 114-117 cm; $431.64 \mathrm{mbsf}$ ), which is consistently lower in $\mathrm{Al}_{2} \mathrm{O}_{3}$ and generally lower in $\mathrm{FeO}(\mathrm{t}), \mathrm{TiO}_{2}$, and $\mathrm{K}_{2} \mathrm{O}$ than the three higher-level samples.

\section{Geochemical Profiles}

Chemical analyses were performed along profiles from each of the investigated samples across microbially altered glass. Each of the altered areas has a central fracture. The analyzed points along the four profiles (Table 3 ) are shown in Plate 5, and the chemical results are in Figure 7. For all the elements, pronounced chemical gradients can be demonstrated. From the fresh basaltic glass to the crystalline material of the central fractures, the four samples show a similar pattern with respect to several elements. Thus, $\mathrm{CaO}$ and $\mathrm{Na}_{2} \mathrm{O}$ get progressively depleted toward the fracture. $\mathrm{SiO}_{2}$ also shows a progressive depletion toward the fracture, but may show a slight enrichment closest to the fresh glass. The pattern shown by $\mathrm{Al}_{2} \mathrm{O}_{3}$ is rather similar to that of $\mathrm{SiO}_{2}$, but the enrichment adjacent to the fresh glass is more pronounced. $\mathrm{TiO}_{2}$ is the most stable element, though it may show some pronounced variations, and is invariably low in the central fracture. With respect to $\mathrm{FeO}(\mathrm{t}), \mathrm{MgO}$, and $\mathrm{K}_{2} \mathrm{O}$, the upper three samples (148- 
Table 2. Microprobe analyses of altered basaltic glass on pillow rims, Hole 896A.

\begin{tabular}{|c|c|c|c|c|c|c|c|c|}
\hline $\begin{array}{l}\text { Core, section, } \\
\text { interval }(\mathrm{cm})\end{array}$ & Form of bodies & $\mathrm{SiO}_{2}$ & $\mathrm{Al}_{2} \mathrm{O}_{3}$ & $\mathrm{TiO}_{2}$ & $\mathrm{FeO}$ & $\mathrm{MgO}$ & $\mathrm{CaO}$ & $\mathrm{Na}_{2} \mathrm{O}$ \\
\hline 148-896A- & & & & & & & & \\
\hline $9 \mathrm{R}-1,17-21$ & Spherical, type I & 49.59 & 15.41 & 0.89 & 8.88 & 7.05 & 9.80 & 1.34 \\
\hline $9 \mathrm{R}-1,17-21$ & Spherical, type I & 50.44 & 16.86 & 0.90 & 7.80 & 8.15 & 4.35 & 0.55 \\
\hline $9 \mathrm{R}-1,17-21$ & Spherical, type I & 50.22 & 16.07 & 0.85 & 8.10 & 7.95 & 6.50 & 0.98 \\
\hline $9 \mathrm{R}-1,17-21$ & Spherical, type I & 51.10 & 16.70 & 0.91 & 7.74 & 7.55 & 5.75 & 0.61 \\
\hline $9 \mathrm{R}-1,17-21$ & Spherical, type I & 50.45 & 16.43 & 0.93 & 7.19 & 7.82 & 5.93 & 0.84 \\
\hline $9 \mathrm{R}-1,17-2 \mathrm{I}$ & Spherical, type I & 49.74 & 16.33 & 0.86 & 7.70 & 8.66 & 5.96 & 0.81 \\
\hline $9 \mathrm{R}-1,17-2 \mathrm{i}$ & Spherical, type I & 41.64 & 14.23 & 0.32 & 11.56 & 10.01 & 1.65 & 0.01 \\
\hline $9 \mathrm{R}-1,17-21$ & Spherical, type I & 49.56 & 15.81 & 0.79 & 7.90 & 7.32 & 8.33 & 1.22 \\
\hline $9 R-1,17-2 i$ & Spherical, type I & 51.77 & 16.76 & 0.91 & 6.78 & 7.68 & 5.43 & 0.68 \\
\hline $9 \mathrm{R}-1,17-21$ & Spherical, type I & 49.03 & 15.63 & 0.85 & 7.95 & 7.73 & 8.59 & 1.29 \\
\hline $9 \mathrm{R}-1,17-21$ & Spherical, type I & 50.11 & 17.09 & 1.03 & 8.90 & 9.00 & 5.42 & 0.71 \\
\hline $9 \mathrm{R}-1,17-21$ & Spherical, type I & 50.63 & 16.59 & 1.05 & 8.24 & 6.53 & 5.56 & 0.62 \\
\hline $9 \mathrm{R}-1,17-21$ & Spherical, type I & 51.58 & 16.54 & 0.90 & 8.81 & 7.80 & 10.26 & 1.45 \\
\hline $9 \mathrm{R}-1.17-21$ & Spherical, type I & 50.44 & 16.54 & 0.87 & 8.58 & 6.80 & 6.78 & 0.96 \\
\hline $9 \mathrm{R}-1,17-2 \mathrm{i}$ & Spherical, type I & 50.21 & 16.39 & 0.87 & 8.69 & 8.21 & 5.50 & 0.78 \\
\hline $9 \mathrm{R}-1,17-21$ & Spherical, type I & 50.33 & 16.14 & 0.90 & 8.40 & 8.29 & 7.57 & 1.27 \\
\hline $9 \mathrm{R}-1,17-21$ & Spherical, type I & 51.82 & 16.60 & 1.01 & 8.33 & 5.53 & 6.34 & 1.07 \\
\hline $9 \mathrm{R}-1,17-2 \mathrm{i}$ & Spherical, type I & 46.18 & 16.02 & 2.20 & 8.58 & 5.18 & 3.58 & 0.75 \\
\hline $9 \mathrm{R}-1,17-21$ & Spherical, type I & 47.36 & 17.02 & 1.41 & 8.25 & 5.07 & 3.52 & 0.26 \\
\hline $9 \mathrm{R}-1.17-21$ & Spherical, type I & 51.35 & 16.11 & 0.83 & 8.89 & 6.75 & 9.20 & 1.41 \\
\hline $9 \mathrm{R}-1,17-21$ & Spherical, type I & 51.16 & 16.03 & 0.93 & 8.65 & 7.88 & 11.58 & 1.67 \\
\hline $9 \mathrm{R}-1,17-2 \mathrm{i}$ & Spherical, type i & 48.78 & 16.89 & 0.91 & 10.03 & 8.05 & 5.06 & 0.69 \\
\hline $9 \mathrm{R}-1,17-2 \mathrm{i}$ & Spherical, type I & 52.35 & 18.26 & 0.86 & 7.08 & 7.79 & 4.60 & 0.58 \\
\hline $9 \mathrm{R}-1,17-21$ & Spherical, type I & 52.11 & 16.28 & 1.02 & 6.80 & 6.29 & 7.58 & 1.10 \\
\hline $9 \mathrm{R}-1,17-21$ & Spherical, type I & 51.84 & 16.73 & 0.87 & 7.53 & 7.32 & 7.49 & 1.06 \\
\hline $11 \mathrm{R}-1,73-75$ & Spherical, type I & 49.87 & 16.86 & 0.95 & 9.47 & 6.99 & 7.76 & 1.18 \\
\hline IIR-1, 73-75 & Spherical, type I & 51.63 & 16.69 & 0.84 & 8.79 & 8.09 & 9.83 & 1.46 \\
\hline IIR-1,73-75 & Spherical, type I & 45.77 & 16.23 & 1.01 & 10.03 & 5.98 & 5.32 & 0.61 \\
\hline IIR-1, 73-75 & Spherical, type I & 49.60 & 16.40 & 0.93 & 8.85 & 7.15 & 7.53 & 1.00 \\
\hline IIR-1.73-75 & Spherical, type I & 50.60 & 16.99 & 0.85 & 8.63 & 6.79 & 6.87 & 0.97 \\
\hline IIR-1,73-75 & Spherical, type I & 48.76 & 16.46 & 1.03 & 10.42 & 6.62 & 8.03 & 1.07 \\
\hline IIR-1, 73-75 & Spherical, type I & 49.81 & 16.54 & 1.09 & 8.94 & 6.63 & 8.29 & 1.23 \\
\hline IIR-1, 73-75 & Spherical, type I & 48.43 & 16.31 & 1.07 & 10.04 & 5.94 & 7.48 & 1.11 \\
\hline IIR-1. 73-75 & Spherical, type I & 49.31 & 16.39 & 0.90 & 9.31 & 5.69 & 6.25 & 0.89 \\
\hline IIR-1, 73-75 & Spherical, type I & 46.34 & 16.13 & 1.48 & 9.54 & 10.20 & 4.97 & 0.88 \\
\hline IIR-1, 73-75 & Spherical, type I & 53.31 & 18.23 & 1.71 & 1.55 & 1.82 & 5.77 & 1.48 \\
\hline IIR-1, 73-75 & Spherical, type I & 53.14 & 17.70 & 1.33 & 2.86 & 2.27 & 6.03 & 1.58 \\
\hline IIR-1, 73-75 & Spherical, type I & 40.46 & 13.93 & 1.40 & 0.90 & 0.69 & 4.77 & 1.14 \\
\hline IIR-1, 73-75 & Spherical, type I & 46.60 & 16.11 & 1.22 & 1.14 & 1.07 & 5.46 & 1.44 \\
\hline IIR-1, 73-75 & Spherical, type I & 46.48 & 17.18 & 0.83 & 11.95 & 9.46 & 4.58 & 0.65 \\
\hline IIR-1.73-75 & Spherical, type i & 49.46 & 17.95 & 0.75 & 8.90 & 8.51 & 4.84 & 0.57 \\
\hline IIR-1.73-75 & Spherical, type I & 49.14 & 17.67 & 0.92 & 7.44 & 6.25 & 5.25 & 0.81 \\
\hline $1 \mid R-1,111-113$ & Spherical, type I & 52.46 & 17.48 & 0.99 & 6.76 & 6.94 & 4.61 & 0.90 \\
\hline IIR-1, $|1|-\mid 13$ & Spherical, type i & 50.47 & 17.10 & 0.86 & 7.94 & 7.51 & 4.86 & 0.75 \\
\hline IIR-1, $111-113$ & Spherical, type I & 49.96 & 17.04 & 1.07 & 7.52 & 5.87 & 3.94 & 0.52 \\
\hline IIR-1, III-113 & Spherical, type I & 50.12 & 18.06 & 1.09 & 8.79 & 6.15 & 4.73 & 0.76 \\
\hline IIR-1, III-113 & Spherical, type I & 47.40 & 17.40 & 0.93 & 8.90 & 5.89 & 3.46 & 0.58 \\
\hline $11 R-1,111-113$ & Spherical, type I & 49.55 & 16.63 & 1.00 & 7.74 & 6.19 & 4.20 & 0.47 \\
\hline IIR-1. III-113 & Spherical, type I & 52.07 & 17.50 & 0.95 & 7.62 & 6.62 & 6.45 & 0.96 \\
\hline $1 \mid R-1,111-113$ & Spherical, type i & 48.47 & 17.19 & 1.07 & 8.65 & 8.06 & 3.28 & 0.45 \\
\hline IIR-1, III-II3 & Spherical, type I & 49.87 & 17.26 & 0.98 & 7.89 & 7.23 & 4.55 & 0.66 \\
\hline$|1 R-1| 1 \mid-113$, & Spherical, type I & 51.35 & 16.72 & $\begin{array}{l}.090 \\
1.03\end{array}$ & 8.31 & 6.00 & 4.70 & 0.62 \\
\hline IIR-1, III-II3 & Spherical, type I & 50.53 & 16.73 & 1.01 & 7.60 & 6.08 & 5.61 & 0.76 \\
\hline $27 \mathrm{R}-1,114-117$ & Spherical, type I & 44.91 & 14.71 & 0.75 & 10.30 & 10.10 & 4.78 & 0.53 \\
\hline $27 \mathrm{R}-1,114-117$ & Spherical, type I & 44.27 & 13.90 & 0.74 & 9.42 & 10.32 & 4.64 & 0.40 \\
\hline $27 \mathrm{R}-1,114-117$ & Spherical, type I & 46.32 & 13.11 & 0.74 & 9.01 & 7.50 & 5.42 & 0.30 \\
\hline $27 \mathrm{R}-1,114-117$ & Spherical, type I & 44.89 & 14.24 & 0.76 & 9.06 & 9.97 & 5.81 & 0.44 \\
\hline $27 \mathrm{R}-1,114-117$ & Spherical, type I & 47.90 & 15.08 & 0.72 & 9.23 & 9.70 & 6.64 & 0.62 \\
\hline $27 \mathrm{R}-1,114-117$ & Spherical, type I & 50.07 & 13.40 & 0.61 & 6.98 & 7.96 & 6.98 & 0.85 \\
\hline $27 \mathrm{R}-1,114-117$ & Spherical, type i & 48.94 & 12.74 & 0.61 & 7.55 & 6.83 & 6.29 & 0.85 \\
\hline $27 \mathrm{R}-1,114-117$ & Spherical, type i & 50.41 & 13.97 & 0.59 & 8.14 & 7.58 & 9.34 & 0.90 \\
\hline $27 \mathrm{R}-1,114-117$ & Spherical, type I & 46.27 & 12.79 & 1.77 & 8.35 & 6.53 & 6.10 & 0.74 \\
\hline $27 R-1,114-117$ & Spherical, type I & 45.45 & 12.82 & 0.66 & 8.74 & 6.73 & 5.97 & 0.91 \\
\hline $27 \mathrm{R}-1,114-117$ & Spherical, type 1 & 43.95 & 12.71 & 1.14 & 8.24 & 7.88 & 4.31 & 0.56 \\
\hline $27 \mathrm{R}-1,114-117$ & Spherical, type i & 47.54 & 13.73 & 0.88 & 8.79 & 8.65 & 4.28 & 0.78 \\
\hline $27 \mathrm{R}-1,114-117$ & Spherical, type 1 & 41.83 & 12.72 & 0.60 & 9.85 & 8.91 & 3.63 & 0.40 \\
\hline $27 \mathrm{R}-1,114-117$ & Spherical, type i & 47.99 & 13.36 & 0.70 & 8.59 & 6.60 & 7.39 & 1.05 \\
\hline $27 \mathrm{R}-\mathrm{I}, 114-117$ & Spherical, type 1 & 48.53 & 13.30 & 0.68 & $\begin{array}{l}8.59 \\
8.01\end{array}$ & $\begin{array}{l}0.00 \\
8.00\end{array}$ & 5.81 & 1.06 \\
\hline IIR-1. 73-75 & Spherical, type 2 & 54.43 & 17.94 & 1.37 & 3.05 & 3.63 & 7.97 & 1.20 \\
\hline & Spherical, type 2 & 49.47 & 17.75 & 1.34 & 3,12 & & 7.81 & 0.86 \\
\hline IIR-I, 73-75 & Spherical, type 2 & 34.53 & 10.79 & 0.63 & 5.30 & 5.05 & 7.32 & $\begin{array}{l}1.00 \\
1.05\end{array}$ \\
\hline IIR-1, 73-75 & Spherical, type 2 & 43.83 & 13.93 & 1.63 & 4.00 & 3.83 & 8.58 & 0.76 \\
\hline IIR-1, 73-75 & Spherical, type 2 & 46.10 & 15.75 & 1.00 & 11.29 & 6.43 & 9.55 & 1.06 \\
\hline IIR-I, 73-75 & Spherical, type 2 & 44.55 & 14.64 & 0.77 & 12.24 & 5.45 & 8.68 & 0.99 \\
\hline IIR-1, 111-113 & Spherical, type 2 & 47.70 & 18.24 & 1.04 & 9.90 & 7.06 & 6.51 & 0.76 \\
\hline$|1 R-1,1| 1 \mid-113$ & Spherical, type 2 & 43.38 & 16.91 & 1.04 & 11.18 & 5.52 & 5.76 & 0.47 \\
\hline IIR-1, III-113 & Spherical, type 2 & 43.96 & 16.46 & 1.06 & 10.24 & 6.08 & 6.47 & 0.66 \\
\hline IIR-1, III-II3 & Spherical, type 2 & 35.29 & 11.19 & 0.99 & 9.35 & 4.36 & 8.66 & 0.57 \\
\hline $\mid 1 R-1,111-113$ & Spherical, type 2 & 46.86 & 17.96 & 0.87 & 10.34 & 6.59 & 6.67 & 0.74 \\
\hline $11 R-1,111-113$ & Spherical, type 2 & 40.92 & 13.49 & 1.02 & 10.25 & 3.95 & 7.42 & 0.74 \\
\hline $1 \mid R-1,111-113$ & Spherical, type 2 & 41.33 & 15.08 & 1.00 & 11.13 & 6.54 & 7.00 & 0.66 \\
\hline $1 \mid R-1,111-113$ & Spherical, type 2 & 44.59 & 15.12 & 0.99 & 10.17 & $\begin{array}{l}0.04 \\
5.14\end{array}$ & 7.76 & 0.66 \\
\hline IIR-1, III-II3 & Spherical, type 2 & 40.79 & 12.92 & 0.99 & 9.54 & 5.20 & 8.83 & 0.61 \\
\hline IIR-I, $111-113$ & Spherical, type 2 & 39.04 & 14.85 & 1.15 & 11.28 & 4.76 & 4.81 & 0.47 \\
\hline IIR-1.1111-113 & Spherical, type 2 & 33.66 & 9.79 & 1.30 & 9.25 & 4.36 & 10.18 & 0.72 \\
\hline $11 R-1,111-113$ & Spherical, type 2 & 43.33 & 15.64 & 1.72 & 12.21 & 4,18 & 5.29 & 1.11 \\
\hline $27 \mathrm{R}-1,114-117$ & Spherical, type 2 & 49.15 & 14.12 & 0.60 & 8.98 & 8.30 & 11.18 & 1.40 \\
\hline $27 \mathrm{R}-1.114-117$ & Spherical, type 2 & 50.75 & 14.69 & 0.63 & 8.11 & 9.03 & 11.76 & 1.45 \\
\hline
\end{tabular}


Table 2 (continued).

\begin{tabular}{|c|c|c|c|c|c|c|c|c|c|}
\hline $\begin{array}{l}\text { Core, section, } \\
\text { interval (cm) }\end{array}$ & Form of bodies & $\mathrm{SiO}_{2}$ & $\mathrm{Al}_{2} \mathrm{O}_{3}$ & $\mathrm{TiO}_{2}$ & $\mathrm{FeO}$ & $\mathrm{MgO}$ & $\mathrm{CaO}$ & $\mathrm{Na}_{2} \mathrm{O}$ & $\mathrm{K}_{2} \mathrm{O}$ \\
\hline 148-896A- & & & & & & & & & \\
\hline $27 \mathrm{R}-1,114-117$ & Spherical, type 2 & 47.72 & 14.44 & 0.62 & 9.00 & 8.37 & 10.68 & 1.20 & 0.24 \\
\hline $27 \mathrm{R}-1.114-117$ & Spherical, type 2 & 45.16 & 13.38 & 0.92 & 9.66 & 7.44 & 8.87 & 0.93 & 0.20 \\
\hline $27 \mathrm{R}-1,114-117$ & Spherical, type 2 & 48.21 & 13.29 & 0.61 & 8.44 & 7.81 & 8.82 & 0.80 & 0.41 \\
\hline $27 \mathrm{R}-1,114-117$ & Spherical, type 2 & 47.63 & 14.29 & 0.46 & 8.12 & 7.79 & 8.45 & 0.68 & 0.40 \\
\hline $27 \mathrm{R}-1,114-117$ & Spherical, type 2 & 46.71 & 13.50 & 0.55 & 8.17 & 7.20 & 9.23 & 0.74 & 0.26 \\
\hline $27 \mathrm{R}-1.114-117$ & Spherical, type 2 & 38.95 & 11.51 & 0.75 & 8.00 & 5.56 & 8.51 & 0.44 & 0.27 \\
\hline $27 \mathrm{R}-1,114-117$ & Spherical, type 2 & 42.93 & 12.73 & 0.46 & 8.22 & 6.82 & 7.86 & 0.53 & 0.39 \\
\hline $27 \mathrm{R}-1,114-117$ & Spherical, type 2 & 42.55 & 11.82 & 0.40 & 8.28 & 5.61 & 8.01 & 0.66 & 0.47 \\
\hline $27 \mathrm{R}-1,114-117$ & Spherical, type 2 & 39.87 & 12.77 & 0.79 & 8.75 & 6.97 & 6.53 & 0.46 & 0.55 \\
\hline $27 \mathrm{R}-1,114-117$ & Spherical, type 2 & 45.29 & 13.05 & 0.57 & 8.79 & 6.66 & 9.23 & 0.92 & 0.51 \\
\hline $27 \mathrm{R}-1,114-117$ & Spherical, type 2 & 41.73 & 11.39 & 0.44 & 8.32 & 5.95 & 7.82 & 0.70 & 0.38 \\
\hline $27 \mathrm{R}-1,114-117$ & Spherical, type 2 & 44.19 & 13.06 & 0.85 & 8.59 & 6.26 & 9.22 & 0.84 & 0.34 \\
\hline $27 \mathrm{R}-1,114-117$ & Spherical, type 2 & 42.12 & 12.08 & 0.61 & 8.66 & 6.12 & 9.70 & 0.75 & 0.41 \\
\hline IIR-I, 73-75 & Spherical, type 3 & 42.92 & 15.46 & 0.81 & 10.79 & 5.35 & 5.38 & 0.60 & 1.10 \\
\hline IIR-1.73-75 & Spherical, type 3 & 41.05 & 15.85 & 1.15 & 11.62 & 4.15 & 3.29 & 0.37 & 1.80 \\
\hline IIR-1.73-75 & Spherical, type 3 & 42.18 & 16.19 & 1.11 & 11.85 & 5.52 & 4.39 & 0.70 & 1.37 \\
\hline IIR-1, 73-75 & Spherical, type 3 & 42.21 & 17.06 & 1.84 & 10.88 & 4.79 & 2.83 & 0.52 & 1.78 \\
\hline IIR-1, 73-75 & Spherical, type 3 & 41.52 & 15.24 & 2.49 & 11.14 & 3.61 & 3.48 & 0.57 & 1.80 \\
\hline IIR-1, 73-75 & Spherical, type 3 & 43.73 & 15.54 & 1.10 & 12.20 & 5.46 & 5.48 & 0.45 & 1.11 \\
\hline IIR-1, 73-75 & Spherical, type 3 & 41.31 & 15.03 & 1.28 & 12.58 & 4.66 & 4.61 & 0.60 & 1.76 \\
\hline IIR-1, 73-75 & Spherical, type 3 & 39.81 & 12.60 & 1.13 & 11.70 & 4.50 & 8.28 & 0.78 & 1.09 \\
\hline IIR-1.73-75 & Spherical, type 3 & 46.28 & 15.91 & 1.12 & 11.17 & 6.23 & 7.50 & 1.09 & 1.10 \\
\hline IIR-1.73-75 & Spherical, type 3 & 42.09 & 13.90 & 1.09 & 12.48 & 4.34 & 5.68 & 0.68 & 1.35 \\
\hline IIR-1, 73-75 & Spherical, type 3 & 39.06 & 16.38 & 1.09 & 10.82 & 4.57 & 3.09 & 0.49 & 1.63 \\
\hline IIR-I. 73-75 & Spherical, type 3 & 41.29 & 15.84 & 1.44 & 10.75 & 4.19 & 3.95 & 0.66 & 1.59 \\
\hline IIR-1, 73-75 & Spherical, type 3 & 51.08 & 16.84 & 1.65 & 3.65 & 2.93 & 7.03 & 1.30 & 2.04 \\
\hline IIR-1, 73-75 & Spherical, type 3 & 50.06 & 18.16 & 2.34 & 0.29 & 0.35 & 5.27 & 2.50 & 4.18 \\
\hline $11 R-1.111-113$ & Spherical, type 3 & 39.08 & 15.43 & 1.18 & 10.97 & 3.66 & 3.41 & 0.34 & 1.81 \\
\hline IIR-1, III-113 & Spherical, type 3 & 43.05 & 16.56 & 2.78 & 11.11 & 3.14 & 4.27 & 0.78 & 1.92 \\
\hline IIR-1, III-113 & Spherical, type 3 & 40.20 & 15.20 & 1.05 & 11.32 & 3.55 & 3.96 & 0.28 & 1.33 \\
\hline IIR-I, III-113 & Spherical, type 3 & 42.53 & 14.64 & 1.20 & 10.55 & 3.46 & 5.85 & 0.49 & 0.99 \\
\hline $11 R-1,111-113$ & Spherical, type 3 & 46.18 & 18.22 & 1.54 & 11.28 & 6.00 & 5.19 & 0.63 & 0.68 \\
\hline $11 \mathrm{R}-1,111-113$ & Spherical, type 3 & 47.32 & 15.32 & 1.36 & 9.54 & 6.24 & 9.80 & 1.12 & 0.56 \\
\hline $11 \mathrm{R}-1,111-113$ & Spherical, type 3 & 41.08 & 14.14 & 0.94 & 9.32 & 6.18 & 8.20 & 0.79 & 0.58 \\
\hline $11 \mathrm{R}-1, \mid 111-113$ & Spherical, type 3 & 39.73 & 12.88 & $\begin{array}{l}1.15 \\
-15\end{array}$ & 9.17 & 3.90 & 7.65 & 0.86 & 0.79 \\
\hline $11 \mathrm{R}-1,111-113$ & Spherical, type 3 & 43.18 & 14.51 & 1.06 & 10.20 & 4.65 & 8.81 & 0.76 & 0.65 \\
\hline $1 \mid \mathrm{R}-1,111-113$ & Spherical, type 3 & 39.40 & 14.35 & 1.19 & 9.88 & 3.67 & 5.81 & 0.47 & 0.65 \\
\hline $11 R-1,111-113$ & Spherical, type 3 & 39.87 & 15.34 & 1.91 & 11.02 & 3.51 & 4.87 & 0.53 & 1.27 \\
\hline IIR-1, $111-113$ & Spherical, type 3 & 40.44 & 14.62 & 1.33 & 11.04 & 3.22 & 4.91 & 0.71 & 1.05 \\
\hline $27 \mathrm{R}-1,114-117$ & Spherical, type 3 & 40.20 & 11.98 & 0.47 & 9.07 & 7.03 & 3.83 & 0.27 & 0.98 \\
\hline $27 \mathrm{R}-1,114-117$ & Spherical, type 3 & 40.17 & 12.81 & 0.42 & 9.98 & 7.65 & 5.78 & 0.42 & 0.53 \\
\hline $27 \mathrm{R}-1,114-117$ & Spherical, type 3 & 36.68 & 9.99 & 0.45 & 9.57 & 4.50 & 3.55 & 0.27 & 0.65 \\
\hline $27 \mathrm{R}-1,114-117$ & Spherical, type 3 & 38.44 & 12.29 & 0.60 & 9.35 & 7.42 & 3.76 & 0.26 & 0.73 \\
\hline $27 \mathrm{R}-1,114-117$ & Spherical, type 3 & 39.55 & 11.45 & 0.50 & 9.01 & 5.98 & 6.01 & 0.33 & 0.84 \\
\hline $27 \mathrm{R}-1,114-117$ & Spherical, type 3 & 41.44 & 11.75 & 0.33 & 8.66 & 6.48 & 6.12 & 0.44 & 0.57 \\
\hline $27 \mathrm{R}-1,114-117$ & Spherical, type 3 & 34.30 & 9.96 & 0.64 & 9.06 & 5.01 & 5.37 & 0.33 & 0.44 \\
\hline $27 \mathrm{R}-1,114-117$ & Spherical, type 3 & 35.64 & 10.94 & 0.90 & 8.87 & 6.06 & 4.46 & 0.22 & 0.46 \\
\hline $27 \mathrm{R}-1,114-117$ & Spherical, type 3 & 38.68 & 11.22 & 0.47 & 8.63 & 7.76 & 4.82 & 0.29 & 0.71 \\
\hline $27 \mathrm{R}-1,114-117$ & Spherical, type 3 & 38.62 & 10.82 & 0.44 & 8.73 & 6.08 & 4.08 & 0.30 & 0.89 \\
\hline $27 \mathrm{R}-1,114-117$ & Spherical, type 3 & 35.97 & 11.17 & 0.52 & 9.04 & 6.20 & 4.17 & 0.34 & 0.74 \\
\hline $27 \mathrm{R}-1,114-117$ & Spherical. type 3 & 39.90 & 11.93 & 0.39 & 9.83 & 5.43 & 4.05 & 0.39 & 0.99 \\
\hline $27 \mathrm{R}-1,114-117$ & Spherical, type 3 & 41.08 & 11.85 & 0.49 & 10.13 & 7.07 & 4.99 & 0.57 & 1.18 \\
\hline $27 \mathrm{R}-1.114-117$ & Spherical, type 3 & 35.21 & 10.13 & 0.54 & 9.04 & 6.24 & 3.57 & 0.28 & 0.91 \\
\hline $27 \mathrm{R}-1,114-117$ & Spherical, type 3 & 34.06 & 9.34 & 0.62 & 9.49 & 4.60 & 5.51 & 0.29 & 0.60 \\
\hline $7 R-1,52-55$ & Vermicular, type 4 & 45.95 & 13.87 & 0.81 & 8.20 & 8.65 & 12.20 & 1.82 & 0.07 \\
\hline $7 \mathrm{R}-1,52-55$ & Vermicular, type 4 & 47.71 & 17.06 & 0.86 & 7.22 & 6.47 & 6.29 & 1.64 & 0.38 \\
\hline $7 \mathrm{R}-1,52-55$ & Vermicular, type 4 & 48.39 & 18.02 & 1.55 & 6.40 & 5.86 & 4.41 & 3.28 & 0.66 \\
\hline $7 \mathrm{R}-1,52-55$ & Vermicular, type 4 & 50.03 & 18.16 & 0.85 & 5.59 & 5.59 & 4.87 & 2.76 & 0.62 \\
\hline $9 \mathrm{R}-1,17-21$ & Vermicular, type 4 & 44.55 & 15.60 & 0.82 & 8.75 & 5.39 & 6.19 & 0.95 & 1.04 \\
\hline $9 \mathrm{R}-1,17-21$ & Vermicular, type 4 & 46.47 & 15.23 & 1.49 & 8.62 & 5.33 & 7.26 & 1.00 & 1.17 \\
\hline $9 \mathrm{R}-1,17-21$ & Vermicular, type 4 & 43.36 & 13.40 & 0.77 & 8.43 & 5.16 & 9.27 & 1.06 & 0.95 \\
\hline $9 \mathrm{R}-1,17-21$ & Vermicular, type 4 & 50.86 & 15.81 & 0.78 & 7.95 & 7.19 & 10.66 & 1.77 & 0.36 \\
\hline $9 \mathrm{R}-1,17-21$ & Vermicular, type 4 & 47.85 & 15.53 & 0.95 & 8.28 & 7.18 & 10.30 & 1.52 & 0.48 \\
\hline $9 \mathrm{R}-1,17-21$ & Vermicular, type 4 & 44.73 & 15.84 & 0.82 & 7.90 & 3.81 & 4.64 & 0.44 & 1.39 \\
\hline $9 \mathrm{R}-1,17-21$ & Vermicular, type 4 & 48.65 & 17.95 & 1.69 & 7.86 & 5.18 & 5.16 & 1.01 & 1.94 \\
\hline $9 \mathrm{R}-1,17-21$ & Vermicular, type 4 & 46.46 & 14.59 & 0.91 & 8.11 & 5.51 & 9.15 & 1.17 & 0.79 \\
\hline $9 \mathrm{R}-1,17-21$ & Vermicular, type 4 & 47.59 & 15.68 & 0.68 & 7.96 & 5.50 & 7.49 & 0.91 & 1.39 \\
\hline IIR-1, 73-75 & Vermicular, type 4 & 43.57 & 16.75 & 1.88 & 10.81 & 3.17 & 2.88 & 0.62 & 1.89 \\
\hline IIR-1,73-75 & Vermicular, type 4 & 45.88 & 15.78 & 0.79 & 10.87 & 4.88 & 6.88 & 0.84 & 1.42 \\
\hline IIR-1.73-75 & Vermicular, type 4 & 42.55 & 17.63 & 2.33 & 10.94 & 4.79 & 2.81 & 0.52 & 2.29 \\
\hline IIR-1.73-75 & Vermicular, type 4 & 44.85 & 16.65 & 1.49 & 10.63 & 4.37 & 4.58 & 0.79 & 2.19 \\
\hline IIR-I, 73-75 & Vermicular, type 4 & 44.55 & 17.81 & 1.58 & 11.43 & 4.00 & 2.99 & 0.57 & 1.85 \\
\hline IIR-1,73-75 & Vermicular, type 4 & 43.41 & 13.94 & 1.01 & 11.50 & 5.06 & 8.09 & 0.95 & 1.03 \\
\hline IIR-1.73-75 & Vermicular, type 4 & 44.66 & 15.48 & 1.14 & 11.31 & 5.39 & 5.28 & 0.84 & 1.21 \\
\hline $11 \mathrm{R}-1,73-75$ & Vermicular, type 4 & 41.40 & 13.41 & 1.05 & 11.65 & 4.27 & 7.98 & 0.95 & 1.05 \\
\hline $11 \mathrm{R}-1,73-75$ & Vermicular, type 4 & 47.56 & 16.05 & 1.08 & 10.54 & 6.17 & 7.64 & 1.17 & 1.31 \\
\hline IIR-1. 73-75 & Vermicular, type 4 & 46.35 & 15.65 & 1.03 & 11.78 & 6.40 & 7.83 & 1.06 & 1.19 \\
\hline IIR-1, 73-75 & Vermicular, type 4 & 42.79 & 18.81 & 2.33 & 11.88 & 5.56 & 3.79 & 0.49 & 1.61 \\
\hline IIR-1, 73-75 & Vermicular, type 4 & 41.14 & 16.89 & 2.06 & 12.62 & 4.26 & 3.34 & 0.59 & 1.56 \\
\hline IIR-1, 73-75 & Vermicular, type 4 & 44.34 & 16.29 & 1.45 & 12.60 & $\begin{array}{l}4.19 \\
\end{array}$ & 4.76 & 0.66 & 1.79 \\
\hline IIR-1,73-75 & Vermicular, type 4 & 44.88 & 16.62 & 0.97 & 13.08 & 4.24 & 5.05 & 0.58 & 1.31 \\
\hline IIR-1, 73-75 & Vermicular, type 4 & 43.04 & 16.00 & 0.95 & 11.95 & 4.57 & 6.05 & 0.68 & 1.19 \\
\hline $11 R-1,111-113$ & Vermicular, type 4 & 43.20 & 17.07 & 1.46 & 11.73 & 5.60 & 5.23 & 0.68 & 0.78 \\
\hline $11 R-1,111-113$ & Vermicular, type 4 & 44.76 & 14.79 & 0.95 & 10.61 & 4.61 & 7.67 & 1.02 & 0.79 \\
\hline $11 R-1,111-113$ & Vermicular, type 4 & 39.69 & 16.79 & 0.95 & 13.18 & 5.76 & 3.03 & 0.28 & 1.00 \\
\hline IIR-I. III-113 & Vermicular, type 4 & 43.63 & 14.96 & 1.06 & 10.26 & 5.21 & 8.47 & 0.98 & 0.73 \\
\hline $11 R-1,111-113$ & Vermicular, type 4 & 44.85 & 17.78 & 1.00 & 11.74 & 6.71 & 5.15 & 0.79 & 0.71 \\
\hline
\end{tabular}


Table 2 (continued).

\begin{tabular}{|c|c|c|c|c|c|c|c|c|c|c|c|c|c|}
\hline $\begin{array}{l}\text { Core, section, } \\
\text { interval }(\mathrm{cm})\end{array}$ & Form of bodies & $\mathrm{SiO}_{2}$ & $\mathrm{Al}_{2} \mathrm{O}_{3}$ & $\mathrm{TiO}_{2}$ & $\mathrm{FeO}$ & $\mathrm{MgO}$ & $\mathrm{CaO}$ & $\mathrm{Na}_{2} \mathrm{O}$ & $\mathrm{K}_{2} \mathrm{O}$ & $\mathrm{MnO}$ & $\mathrm{P}_{2} \mathrm{O}_{5}$ & $\mathrm{Cr}_{2} \mathrm{O}_{5}$ & Total \\
\hline \multicolumn{14}{|l|}{ 148-896A- } \\
\hline $11 R-1,111-113$ & Vermicular, type 4 & 41.13 & 18.30 & 1.84 & 12.86 & 4.82 & 3.21 & 0.58 & 1.33 & 0.25 & ND & ND & 84.32 \\
\hline $11 \mathrm{R}-1.111-113$ & Vermicular, type 4 & 39.62 & 16.87 & 1.13 & 13.18 & 4.73 & 3.05 & 0.27 & 1.16 & 0.30 & ND & ND & 80.31 \\
\hline $11 R-1,111-113$ & Vermicular, type 4 & 45.48 & 16.33 & 1.45 & 12.27 & 4.62 & 6.09 & 0.82 & 1.14 & 0.19 & ND & ND & 88.39 \\
\hline $11 \mathrm{R}-1.111-113$ & Vermicular, type 4 & 43.40 & 16.90 & 1.86 & 11.50 & 4.08 & 4.32 & 0.69 & 1.59 & 0.10 & ND & ND & 84.44 \\
\hline $11 R-1,111-113$ & Vermicular, type 4 & 46.69 & 16.68 & 1.08 & 11.39 & 5.55 & 7.89 & 0.87 & 1.03 & 0.26 & ND & ND & 91.44 \\
\hline $11 R-1,111-113$ & Vermicular, type 4 & 45.95 & 15.43 & 1.09 & 10.80 & 6.34 & 9.80 & 1.09 & 0.57 & 0.12 & ND & ND & 91.19 \\
\hline $11 R-1,111-113$ & Vermicular, type 4 & 44.58 & 16.04 & 1.56 & 11.33 & 4.49 & 6.18 & 1.19 & 0.74 & 0.11 & ND & ND & 86.23 \\
\hline $27 \mathrm{R}-1,114-117$ & Vermicular, type 4 & 46.46 & 13.35 & 0.85 & 9.72 & 7.64 & 6.48 & 0.58 & 0.71 & 0.17 & ND & ND & 85.96 \\
\hline $27 \mathrm{R}-1,114-117$ & Vermicular, type 4 & 46.32 & 14.43 & 0.55 & 8.85 & 8.98 & 9.71 & 1.22 & 0.37 & 0.28 & ND & ND & 90.71 \\
\hline $27 \mathrm{R}-1,114-117$ & Vermicular, type 4 & 50.70 & 16.04 & 0.56 & 9.05 & 9.62 & 11.73 & 1.32 & 0.24 & 0.25 & ND & ND & 99.51 \\
\hline $27 \mathrm{R}-1,114-117$ & Vermicular, type 4 & 49.18 & 14.85 & 0.63 & 9.80 & 9.15 & 9.46 & 1.25 & 0.41 & 0.13 & ND & ND & 94.86 \\
\hline $27 \mathrm{R}-1,114-117$ & Vermicular, type 4 & 41.19 & 12.95 & 0.89 & 9.70 & 7.47 & 4.27 & 0.31 & 0.55 & 0.29 & ND & ND & 77.62 \\
\hline $27 \mathrm{R}-1,114-117$ & Vermicular, type 4 & 42.67 & 13.83 & 0.57 & 10.62 & 7.81 & 5.16 & 0.42 & 0.78 & 0.30 & ND & ND & 82.16 \\
\hline $27 \mathrm{R}-1,114-117$ & Vermicular, type 4 & 38.59 & 11.78 & 0.38 & 9.17 & 6.37 & 4.31 & 0.39 & 0.71 & 0.22 & ND & ND & 71.92 \\
\hline $27 \mathrm{R}-1,114-117$ & Vermicular, type 4 & 39.68 & 12.48 & 0.73 & 9.65 & 6.78 & 5.72 & 0.32 & 0.67 & 0.21 & ND & ND & 76.24 \\
\hline $27 \mathrm{R}-1,114-117$ & Vermicular, type 4 & 42.17 & 12.31 & 0.66 & 8.50 & 6.34 & 9.79 & 0.82 & 0.40 & 0.19 & ND & ND & 81.18 \\
\hline $27 \mathrm{R}-1,114-117$ & Vermicular, type 4 & 44.58 & 13.31 & 0.40 & 9.96 & 7.36 & 4.55 & 0.34 & 0.93 & 0.14 & ND & ND & 81.57 \\
\hline $27 \mathrm{R}-1,114-117$ & Vermicular, type 4 & 40.78 & 12.07 & 0.66 & 8.29 & 6.83 & 6.43 & 0.50 & 0.82 & 0.11 & ND & ND & 76.49 \\
\hline $27 \mathrm{R}-1,114-117$ & Vermicular, type 4 & 37.26 & 11.52 & 0.54 & 9.29 & 5.74 & 6.53 & 0.41 & 0.59 & 0.27 & ND & ND & 72.15 \\
\hline $27 \mathrm{R}-1.114-117$ & Vermicular, type 4 & 36.48 & 12.11 & 0.26 & 8.57 & 7.06 & 4.18 & 0.18 & 0.44 & 0.19 & ND & ND & 69.47 \\
\hline $27 \mathrm{R}-1,114-117$ & Vermicular, type 4 & 41.46 & 12.60 & 0.44 & 8.22 & 6.66 & 7.36 & 0.58 & 0.50 & 0.15 & ND & ND & 77.97 \\
\hline $27 \mathrm{R}-1,114-117$ & Vermicular, type 4 & 43.43 & 12.63 & 0.79 & 9.41 & 6.27 & 7.73 & 0.60 & 0.41 & 0.03 & ND & ND & 81.30 \\
\hline
\end{tabular}

Notes: $\mathrm{FeO}(\mathrm{t})=$ total iron reported as $\mathrm{FeO}, \mathrm{ND}=$ not determined.

Table 3. Microprobe analyses of altered basaltic glass along four profiles across veins, Hole 896A.

\begin{tabular}{|c|c|c|c|c|c|c|c|c|c|c|c|}
\hline $\begin{array}{l}\text { Core, section, } \\
\text { interval }(\mathrm{cm})\end{array}$ & $\mathrm{N}$ & $\mathrm{SiO}_{2}$ & $\mathrm{Al}_{2} \mathrm{O}_{3}$ & $\mathrm{TiO}_{2}$ & $\mathrm{FeO}$ & $\mathrm{MgO}$ & $\mathrm{CaO}$ & $\mathrm{Na}_{2} \mathrm{O}$ & $\mathrm{K}_{2} \mathrm{O}$ & $\mathrm{MnO}$ & Total \\
\hline \multicolumn{12}{|l|}{ 148-896A- } \\
\hline $9 \mathrm{R}-1,17-21$ & 1 & 52.18 & 17.45 & 0.96 & 6.98 & 5.94 & 6.50 & 1.26 & 1.00 & 0.22 & 92.49 \\
\hline $9 R-1,17-21$ & 2 & 48.29 & 18.08 & 0.61 & 10.43 & 9.73 & 3.60 & 0.41 & 0.80 & 0.48 & 92.43 \\
\hline $9 \mathrm{R}-\mathrm{I}, 17-21$ & 3 & 41.08 & 16.56 & 0.67 & 10.93 & 9.01 & 1.87 & 0.09 & 0.42 & 0.23 & 80.86 \\
\hline $9 \mathrm{R}-1.17-21$ & 4 & 40.27 & 16.22 & 0.62 & 12.80 & 11.31 & 1.69 & 0.08 & 0.36 & 0.51 & 83.80 \\
\hline $9 \mathrm{R}-1,17-21$ & 5 & 37.12 & 12.73 & 0.15 & 15.65 & 10.74 & 1.51 & 0.06 & 0.54 & 0.37 & 78.22 \\
\hline $9 \mathrm{R}-1,17-21$ & 6 & 47.56 & 17.19 & 0.78 & 9.49 & 9.60 & 2.47 & 0.14 & 0.76 & 0.30 & 88.29 \\
\hline $9 \mathrm{R}-1,17-21$ & 7 & 48.46 & 17.50 & 0.88 & 8.11 & 7.03 & 2.86 & 0.25 & 1.03 & 0.35 & 86.47 \\
\hline $9 \mathrm{R}-1,17-21$ & 8 & 48.43 & 17.31 & 1.08 & 6.94 & 6.93 & 3.08 & 0.32 & 0.95 & 0.14 & 85.18 \\
\hline $9 \mathrm{R}-1,17-21$ & 9 & 51.20 & 16.88 & 0.84 & 8.18 & 7.00 & 9.00 & 1.29 & 0.70 & 0.09 & 95.18 \\
\hline IIR-1, 73-75 & 1 & 49.62 & 16.38 & 0.96 & 9.43 & 6.81 & 6.83 & 0.85 & 0.90 & 0.14 & 91.92 \\
\hline IIR-1, 73-75 & 2 & 50.98 & 16.08 & 0.84 & 9.23 & 7.41 & 6.42 & 0.99 & 0.93 & 0.13 & 93.01 \\
\hline IIR-I. 73-75 & 3 & 44.36 & 14.72 & 1.10 & 11.29 & 10.09 & 2.14 & 0.25 & 0.89 & 0.27 & 85.11 \\
\hline IIR-1.73-75 & 4 & 39.03 & 14.23 & 0.38 & 13.85 & 10.98 & 1.93 & 0.08 & 0.46 & 0.49 & 81.43 \\
\hline IIR-1, 73-75 & 5 & 39.27 & 15.23 & 1.03 & 13.45 & 10.95 & 1.89 & 0.12 & 0.71 & 0.64 & 83.29 \\
\hline IIR-1, 73-75 & 6 & 40.78 & 14.83 & 0.12 & 12.18 & 10.75 & 2.01 & 0.41 & 0.87 & 0.45 & 82.40 \\
\hline IIR-1,73-75 & 7 & 40.15 & 14.36 & 0.10 & 12.13 & 9.17 & 1.90 & 0.26 & 0.82 & 0.41 & 79.30 \\
\hline $11 \mathrm{R}-1,73-75$ & 8 & 41.67 & 13.77 & 1.12 & 11.86 & 10.82 & 1.77 & 0.10 & 0.70 & 0.08 & 81.89 \\
\hline $11 \mathrm{R}-1.73-75$ & 9 & 45.72 & 15.46 & 0.92 & 10.59 & 10.32 & 2.17 & 0.15 & 0.83 & 0.45 & 86.61 \\
\hline IIR-1, 73-75 & 10 & 49.53 & 15.96 & 1.19 & 9.76 & 7.68 & 4.39 & 0.45 & 1.20 & 0.15 & 90.31 \\
\hline IIR-I, 73-75 & 11 & 46.36 & 15.42 & 1.15 & 10.96 & 5.63 & 7.49 & 0.85 & 1.21 & 0.25 & 89.32 \\
\hline $11 R-1,111-113$ & 1 & 52.74 & 17.44 & 0.81 & 7.68 & 6.98 & 6.92 & 1.12 & 0.90 & 0.25 & 94.84 \\
\hline $11 R-1,111-113$ & 2 & 49.79 & 17.57 & 0.91 & 7.84 & 7.24 & 3.43 & 0.48 & 0.92 & 0.22 & 88.40 \\
\hline $11 \mathrm{R}-1,111-113$ & 3 & 47.59 & 15.99 & 0.94 & 8.30 & 8.24 & 2.56 & 0.25 & 0.79 & 0.10 & 84.76 \\
\hline $11 R-1,111-113$ & 4 & 40.88 & 15.55 & 0.81 & 13.28 & 11.42 & 1.91 & 0.07 & 0.41 & 0.57 & 84.90 \\
\hline $11 R-1,111-113$ & 5 & 39.25 & 13.41 & 0.33 & 14.78 & 10.87 & 1.65 & 0.04 & 0.55 & 0.33 & 81.21 \\
\hline $11 R-1,111-113$ & 6 & 39.65 & 14.49 & 0.58 & 14.30 & 12.41 & 1.74 & 0.04 & 0.40 & 0.47 & 84.08 \\
\hline $11 R-1,111-113$ & 7 & 41.58 & 15.52 & 0.95 & 10.65 & 10.31 & 2.12 & 0.18 & 0.72 & 0.36 & 82.39 \\
\hline $11 \mathrm{R}-1,111-113$ & 8 & 50.81 & 16.28 & 0.94 & 7.39 & 6.67 & 4.72 & 0.62 & 0.85 & 0.21 & 88.49 \\
\hline $11 R-1,111-113$ & 9 & 50.28 & 17.18 & 0.96 & 8.71 & 6.95 & 7.85 & 0.98 & 0.59 & 0.17 & 93.67 \\
\hline $27 \mathrm{R}-1,114-117$ & 1 & 44.91 & 16.31 & 0.34 & 10.11 & 11.53 & 4.71 & 0.52 & 0.33 & 0.34 & 89.10 \\
\hline $27 \mathrm{R}-1,114-117$ & 2 & 42.13 & 15.48 & 0.61 & 11.42 & 11.55 & 3.87 & 0.19 & 0.34 & 0.32 & 85.91 \\
\hline $27 \mathrm{R}-1,114-117$ & 3 & 43.05 & 14.56 & 1.13 & 9.23 & 10.54 & 4.22 & 0.08 & 0.37 & 0.13 & 83.37 \\
\hline $27 \mathrm{R}-1,114-117$ & 4 & 43.43 & 13.99 & 1.24 & 9.13 & 9.56 & 4.26 & 0.10 & 0.43 & 0.18 & 82.32 \\
\hline $27 \mathrm{R}-1,114-117$ & 5 & 41.03 & 11.62 & 0.50 & 13.10 & 5.97 & 3.19 & 0.05 & 1.25 & 0.12 & 76.83 \\
\hline $27 \mathrm{R}-1,114-117$ & 6 & 42.42 & 13.49 & 0.96 & 8.16 & 8.45 & 4.13 & 0.11 & 0.50 & 0.19 & 78.41 \\
\hline $27 \mathrm{R}-1,114-117$ & 7 & 42.69 & 13.75 & 1.42 & 8.95 & 8.47 & 4.26 & 0.05 & 0.40 & 0.20 & 80.19 \\
\hline $27 \mathrm{R}-1,114-117$ & 8 & 48.22 & 15.13 & 0.64 & 7.07 & 8.41 & 4.91 & 0.25 & 0.76 & 0.31 & 85.70 \\
\hline $27 \mathrm{R}-1,114-117$ & 9 & 50.84 & 14.78 & 0.46 & 7.72 & 8.10 & 8.28 & 0.83 & 0.45 & 0.32 & 91.78 \\
\hline
\end{tabular}

Notes: $\mathrm{FeO}(\mathrm{t})=$ total iron reported as $\mathrm{FeO}$.

896A-9R-1, 17-21 cm; 11R-1, 73-75 cm; and 11R-1, 111-113 cm) show consistently similar patterns that differ from that of the lowest sample (148-896A-27R-1, 114-117 cm). Thus, FeO(t) and $\mathrm{MgO}$ for the upper three samples are depleted adjacent to the fresh glass and increase progressively toward the fracture. With respect to the deepest sample, $\mathrm{FeO}(\mathrm{t})$ and $\mathrm{MgO}$ do not show consistent depletion adjacent to the fresh glass, and $\mathrm{MgO}$ shows the lowest concentration in the fracture. $\mathrm{K}_{2} \mathrm{O}$ shows, for the upper three samples, a consistent pattern in which the highest concentration is closest to the fresh glass with concentration decreasing toward the fracture. For the deepest sample, however, $\mathrm{K}_{2} \mathrm{O}$ is generally lower in the altered glass and is highest in the fracture.

\section{DISCUSSION}

Some crucial questions in this connection are as follows: (1) are bacteria especially attracted by the basaltic glass; (2) how do they dissolve it; and ( 3 ) what important chemical function do they play? 


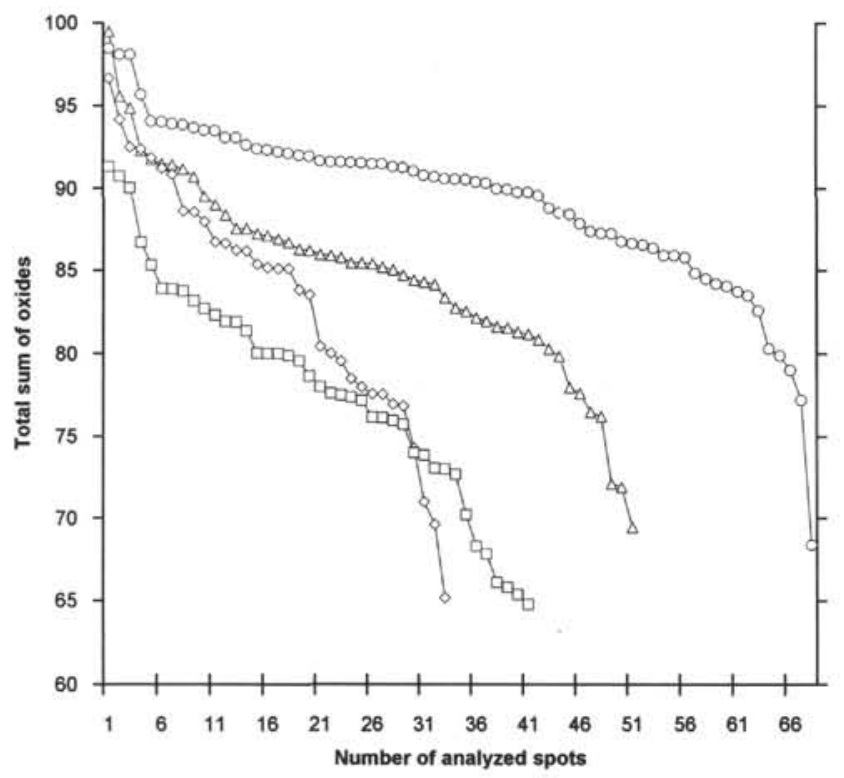

Figure 1. Diagram showing the total sum of oxides and the number of analyzed spots of the various types of alteration morphologies. Circles = type 1, squares $=$ type 2 , diamonds $=$ type 3 , and triangles $=$ type 4 .
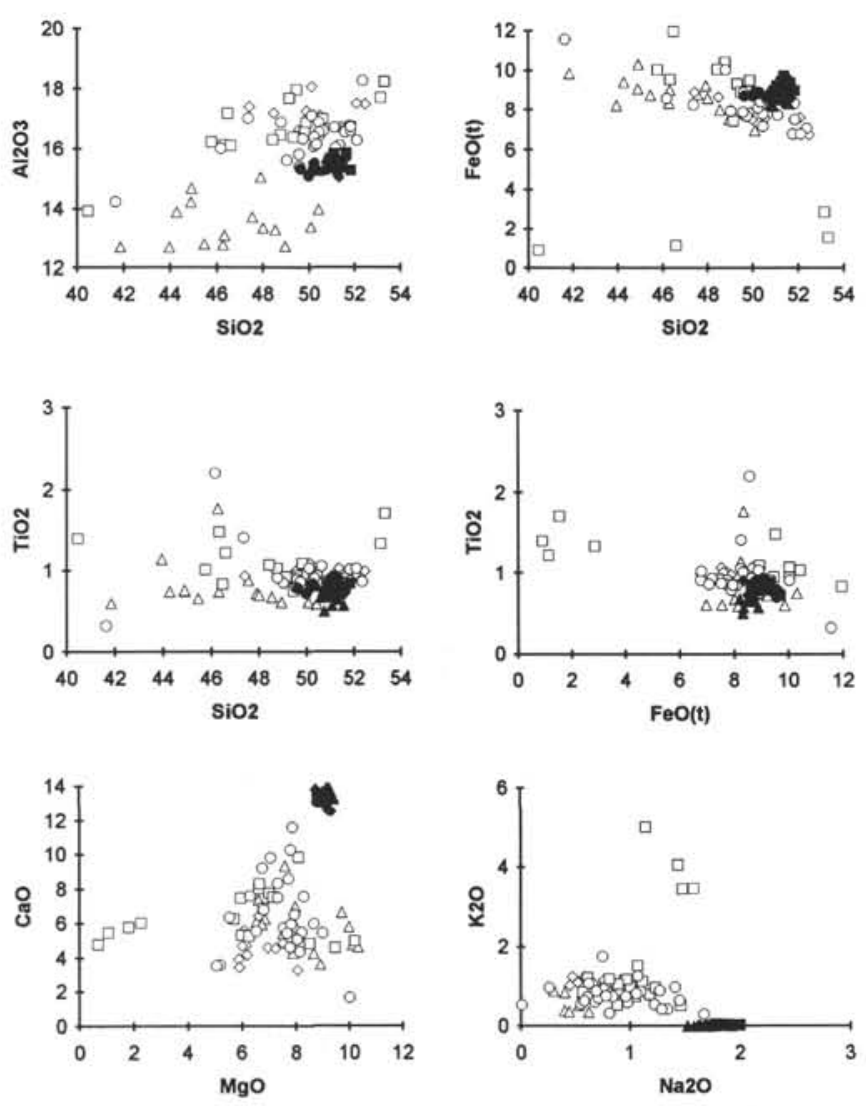

Figure 2. $\mathrm{Al}_{2} \mathrm{O}_{3} / \mathrm{SiO}_{2}, \mathrm{FeO}(\mathrm{t}) / \mathrm{SiO}_{2}, \mathrm{TiO}_{2} / \mathrm{SiO}_{2}, \mathrm{TiO}_{2} / \mathrm{FeO}(\mathrm{t}), \mathrm{CaO} / \mathrm{MgO}$, and $\mathrm{K}_{2} \mathrm{O} / \mathrm{Na}_{2} \mathrm{O}$ relations for microbially processed glass (open symbols) of the type 1 morphology compared to fresh basaltic glass (solid symbols). Circles = Sample 148-896A-9R-1, 17-21 cm; squares $=$ Sample 148-896A-1IR-1. $73-75 \mathrm{~cm}$; diamonds $=$ Sample $148-896 \mathrm{~A}-11 \mathrm{R}-1,111-113 \mathrm{~cm}$; and triangles $=$ Sample 148-896A-27R-1, 114-117 cm.
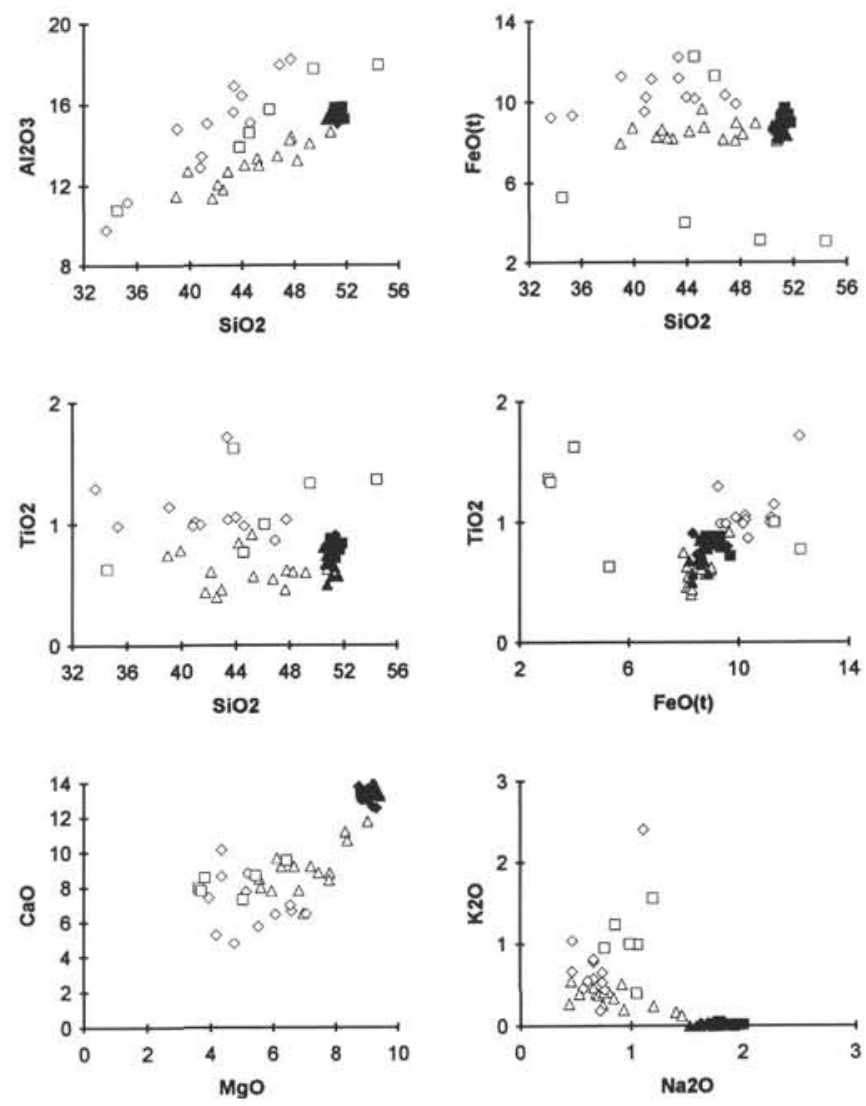

Figure 3. $\mathrm{Al}_{2} \mathrm{O}_{3} / \mathrm{SiO}_{2}, \mathrm{FeO}(\mathrm{t}) / \mathrm{SiO}_{2}, \mathrm{TiO}_{2} / \mathrm{SiO}_{2}, \mathrm{TiO}_{2} / \mathrm{FeO}(\mathrm{t}), \mathrm{CaO} / \mathrm{MgO}$, and $\mathrm{K}_{2} \mathrm{O} / \mathrm{Na}_{2} \mathrm{O}$ relations for microbially processed glass (open symbols) of the type 2 morphology compared to fresh basaltic glass (solid symbols). Circles $=$ Sample 148-896A-9R-1, 17-21 cm; squares = Sample 148-896A-11R-1, $73-75 \mathrm{~cm}$; diamonds $=$ Sample $148-896 \mathrm{~A}-11 \mathrm{R}-1,111-113 \mathrm{~cm}$; and triangles $=$ Sample 148-896A-27R-1, 114-117 cm.

\section{Bacteria-Basaltic Glass Problem}

Simple experiments in which granular, slightly altered basaltic tuff is subjected to bacteria-contaminated distilled water show that the bacteria to a large extent prefer settlement on the fresh glass particles. Three different models may be proposed. First, glass surfaces may serve as binding sites for organic material that could serve as substrate for bacterial metabolism (Krumbein, 1983; Ehrlich, 1981). Second, glass contains elements that could serve as electron acceptors in a respiration process. Bacteria from a depth of $400 \mathrm{mbsf}$, retrieved from below $200 \mathrm{~m}$ of marine sediments, probably have an anaerobic metabolism. Possible electron acceptors would be Fe or Mn (Jannasch and Mottl, 1985). Third, glass contains crystallization energy, which, if released in a biologically useful way, could serve as an energy source for bacterial metabolism.

The textural as well as chemical development of basaltic glass surfaces has been demonstrated by previous studies on natural glass (e.g. Thorseth et al., 1991; Thorseth et al., 1992a, 1992b) and by experimental studies (Berger et al., 1987; Crovisier et al., 1987; Gislason and Eugster, 1987; Guy and Schott, 1989; Thorseth et al., in press) that demonstrate clearly that the glass materials may dissolve by various mechanisms.

To dissolve the glass congruently, its $\mathrm{Si}$ and $\mathrm{Al}$ networks have to be destroyed. This phenomenon may occur over a large $\mathrm{pH}$ range, from nearly neutral to alkaline conditions, but to achieve a high rate of dissolution, a pH $>9$ is required (Guy and Schott, 1989). At acidic 

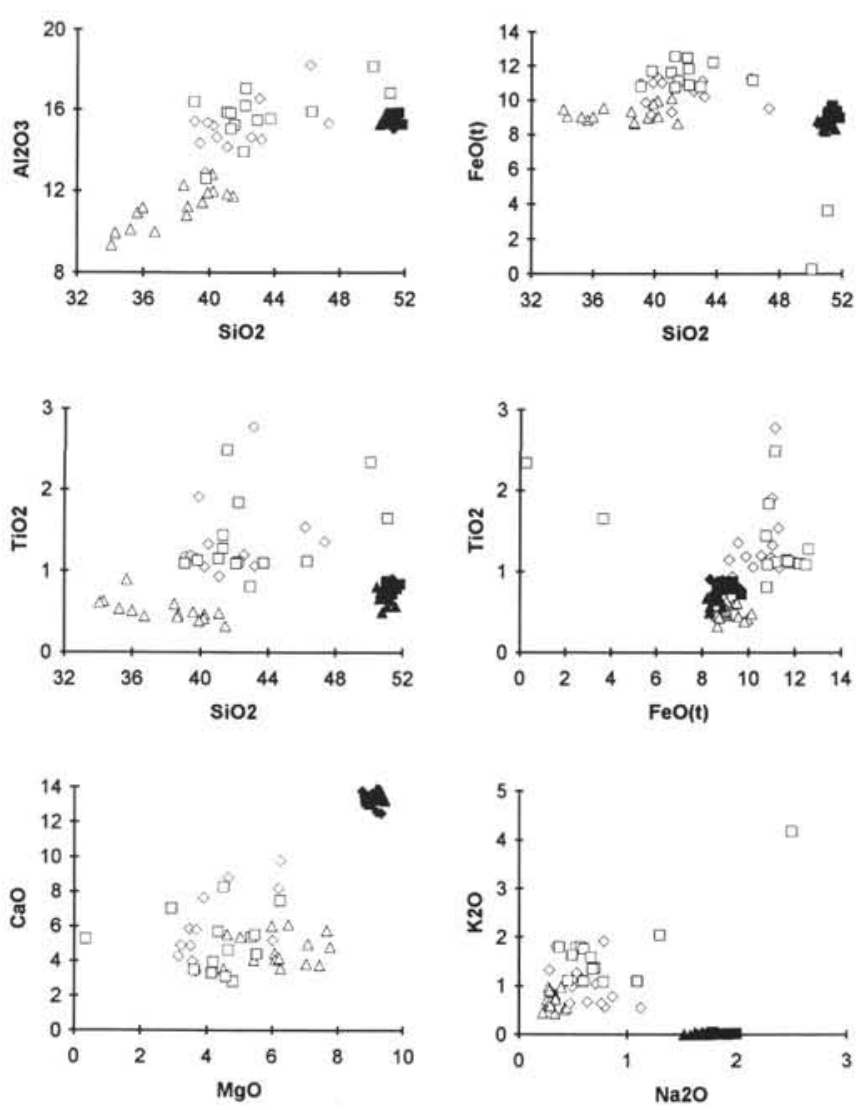

Figure 4. $\mathrm{Al}_{2} \mathrm{O}_{3} / \mathrm{SiO}_{2}, \mathrm{FeO}(\mathrm{t}) / \mathrm{SiO}_{2}, \mathrm{TiO}_{2} / \mathrm{SiO}_{2}, \mathrm{TiO}_{2} / \mathrm{FeO}(\mathrm{t}), \mathrm{CaO} / \mathrm{MgO}$, and $\mathrm{K}_{2} \mathrm{O} / \mathrm{Na}_{2} \mathrm{O}$ relations for microbially processed glass (open symbols) of the type 3 morphology compared to fresh basaltic glass (solid symbols). Circles = Sample 148-896A-9R-1, 17-21 cm; squares $=$ Sample 148-896A-11R-1, $73-75 \mathrm{~cm}$; diamonds $=$ Sample $148-896 \mathrm{~A}-1 \mathrm{IR}-1,111-113 \mathrm{~cm}$; and triangles $=$ Sample 148-896A-27R-1, 114-117 cm.

conditions, the glass dissolves incongruently by selective leaching of $\mathrm{Fe}, \mathrm{Al}$, and $\mathrm{Ti}$ (Paul and Zaman, 1978). Local $\mathrm{pH}$ conditions will be achieved in contact with, or adjacent to, bacteria (Golubic, 1973; Peschek, 1987). In an experimental approach to determine the extent to which bacteria may dissolve basaltic glass, two different stages were discovered (Thorseth et al., in press). The first mechanism encompasses total dissolution of the glass, resulting in a biopitted surface, followed by selective leaching of all elements from the glass except $\mathrm{Si}$, which are suggested to take place at high and low $\mathrm{pH}$, respectively.

Thus, one may suggest that the micropits and microchannels that define the four alteration-generated bodies (types 1-4; Pls. 2, 3) formed as a result of microbial dissolution of the basalt glass. However, the four different types of bodies are heterogeneous with respect to all oxides (Figs. 2-6). As discussed below, these chemical features may, by applying the results of the experiment of Thorseth et al. (in press), be the result of microbial processes.

\section{Where Can Microbes Exist?}

Observations of bacterial activity in aquifers and deep oil wells (Straube et al., 1990; Rosnes et al., 1991; Bernard et al., 1992; Hiebert and Bennett, 1992) have triggered discussion as to whether bacteria are indigenous or introduced during sampling or by the injection of water (Deming and Baross, 1993; Parkes and Maxwell, 1993; Stetter et al., 1993). Microbes live in high-temperature regions in the lithosphere, such as oil reservoirs, aquifers, iron-rich ores, ocean
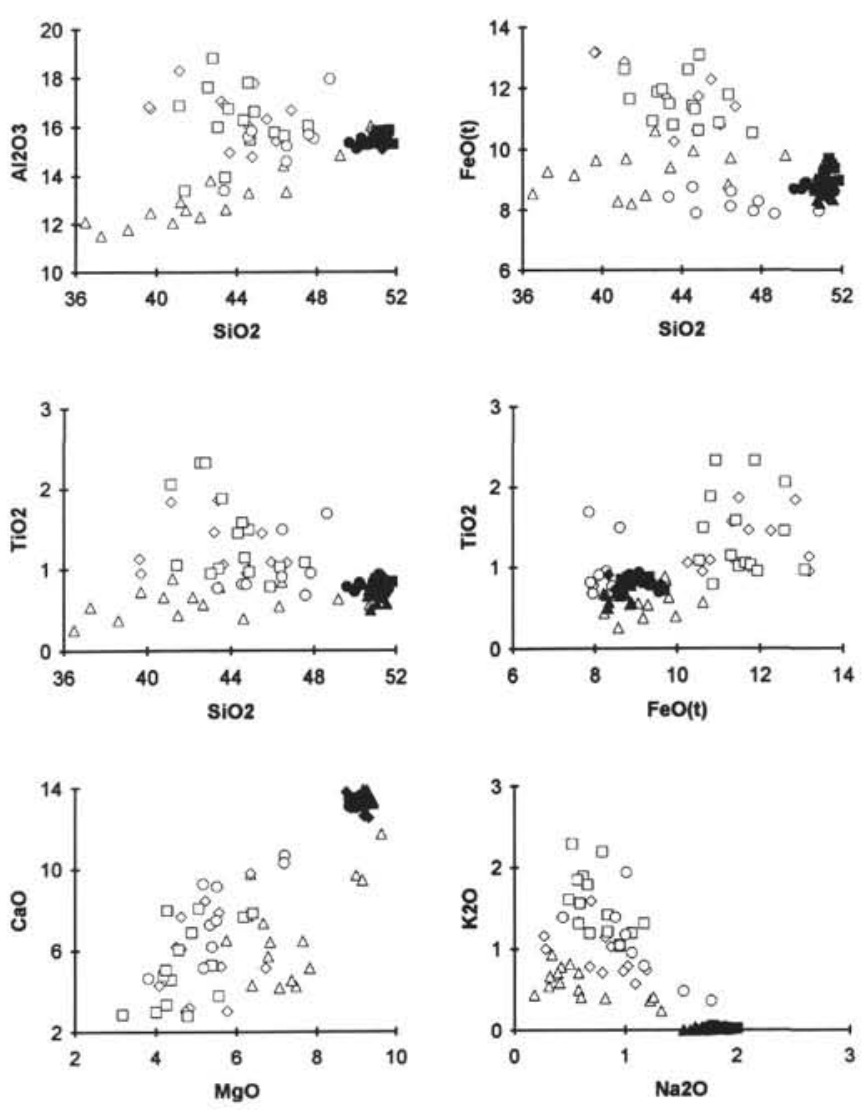

Figure 5. $\mathrm{Al}_{2} \mathrm{O}_{3} / \mathrm{SiO}_{2}, \mathrm{FeO}(\mathrm{t}) / \mathrm{SiO}_{2}, \mathrm{TiO}_{2} / \mathrm{SiO}_{2}, \mathrm{TiO}_{2} / \mathrm{FeO}(\mathrm{t}), \mathrm{CaO} / \mathrm{MgO}$, and $\mathrm{K}_{2} \mathrm{O} / \mathrm{Na}_{2} \mathrm{O}$ relations for microbially processed glass (open symbols) of the type 4 morphology compared to fresh basaltic glass (solid symbols). Circles $=$ Sample 148-896A-9R-1, 17-21 cm; squares $=$ Sample 148-896A-11R-1, $73-75 \mathrm{~cm}$; diamonds $=$ Sample $148-896 \mathrm{~A}-11 \mathrm{R}-1,111-113 \mathrm{~cm}$; and triangles $=$ Sample 148-896A-27R-1, 114-117 cm.

rifts, and hot springs (Brock, 1985). Bacteria are known to grow at $110^{\circ} \mathrm{C}$ (Huber et al., 1989) and up to 1000 bar (Jannasch and Taylor, 1984). The definite limit of life is still unknown. Brock (1985) suggested $150^{\circ} \mathrm{C}$ as an upper limit, whereas others would accept temperatures approaching $400^{\circ} \mathrm{C}$ at abyssal depths (Deming and Baross, 1993). In large parts of the upper oceanic crust containing pillow lavas, temperature would not limit microbial activity. On the basis of the textural relationships and the DAPI staining (Pls. 1, 2), we conclude that microbes, at least $237 \mathrm{~m}$ deep in the volcanic basement of the oceanic crust at a temperature of about $70^{\circ} \mathrm{C}$ (Shipboard Scientific Party, 1993), have attached to the basaltic glass, accelerated its dissolution at the glass/cell interface, and resulted in the formation of narrow channels in which they live. These observations establish the existence of a subterranean biosphere.

\section{Geochemistry Controlled by Microbes}

We have shown that microbes attach to basaltic glass surfaces and cause dissolution (Thorseth et al., in press). To account for the chemical trends shown in Figures 2 through 6, we suggest that they most probably can be attributed to microbial processes. Important in this respect is the experiment in which basaltic glass was immersed in growth media inoculated with mixed cultures of bacteria enriched from Surtsey tephra (Thorseth et al., in press). The experiment showed that the cell walls of bacteria attached to the glass and accumulated $\mathrm{Si}$ and $\mathrm{Al}$. The concentration of the elements may vary con- 

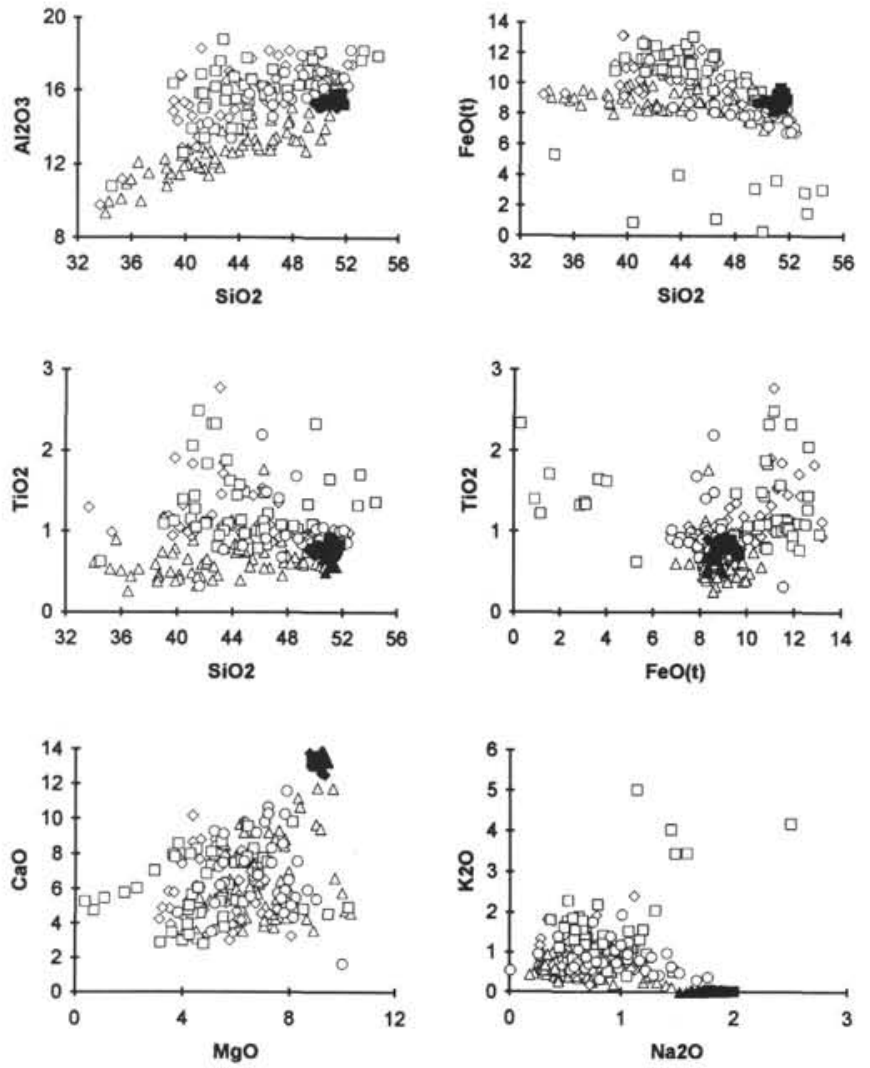

Figure 6. $\mathrm{Al}_{2} \mathrm{O}_{3} / \mathrm{SiO}_{2}, \mathrm{FeO}(\mathrm{t}) / \mathrm{SiO}_{2}, \mathrm{TiO}_{2} / \mathrm{SiO}_{2}, \mathrm{TiO}_{2} / \mathrm{FeO}(\mathrm{t}), \mathrm{CaO} / \mathrm{MgO}$, and $\mathrm{K}_{2} \mathrm{O} / \mathrm{Na}_{2} \mathrm{O}$ relations for microbially processed glass (open symbols) compared to fresh basaltic glass (filled symbols). Each symbol represents all four types at a specific depth. Circles $=$ Sample 148-896A-9R-1. 17-21 cm; squares $=$ Sample 148-896A-11R-1, 73-75 cm; diamonds $=$ Sample $148-$ $896 \mathrm{~A}-11 \mathrm{R}-1,111-113 \mathrm{~cm}$; and triangles = Sample 148-896A-27R-1, 114$117 \mathrm{~cm}$.

siderably, probably depending on the variety of bacteria. Other elements accumulated are $\mathrm{Mg}, \mathrm{Ca}, \mathrm{Fe}, \mathrm{K}$, and $\mathrm{P}$. A study by Ferris et al. (1986) showed that thermophilic bacteria living at approximately $60^{\circ} \mathrm{C}$ in hot springs appear to provide the nucleation sites for iron-silica phases. Similarly, iron oxide/silica materials from the seafloor at several spreading ridges are porous and consist of branching filaments (Juniper and Fouquet, 1988).

A recent observation is the identification of nannobacteria $(0.05$ to $0.2 \mu \mathrm{m}$ in diameter) occurring in different natural environments and their ability to cause precipitation of a large number of components such as silica and silicates and iron oxides (Folk, 1994). These may be comparable in size to those microbes responsible for the type 1 alteration textures. However, the concentrations of $\mathrm{SiO}_{2}, \mathrm{Al}_{2} \mathrm{O}_{3}$, $\mathrm{TiO}_{2}$, and $\mathrm{FeO}(\mathrm{t})$ within the microchannels are roughly similar to that of the adjacent glass (Figs. 2-5). We think that the most effective way of accumulating $\mathrm{Si}, \mathrm{Al}$, and $\mathrm{Fe}$ is by precipitation in capsular material surrounding the cell wall, whereas $\mathrm{Ca}$ and $\mathrm{Mg}$ are lost. The high concentration of $\mathrm{K}_{2} \mathrm{O}$ (mostly between 1 and 2 wt\%; Figs. 2-6) within the microbially processed areas is a property that can be attributed to viable cells (Harder and Dijkhuisen, 1983). Furthermore, these high concentrations were not derived from the host basalt glass, as they had an average $\mathrm{K}_{2} \mathrm{O}$ content of about 0.03 wt\% (Shipboard Scientific Party, 1993). The only possible source for K is seawater, of which large amounts would have to circulate through the rocks.

\section{Consequences and Dimensions of Microbial Activity}

What are the possible consequences and dimensions of the microbial activity on the basaltic glass in terms of geochemical budgets in the oceanic crust and the ocean water? In this respect, it is appropriate to first make some primitive estimates on (I) the volume and surface area of basaltic glass within the oceanic crust and (2) the rate of microbial processing.

\section{Volume and Surface Area of Basaltic Glass}

Concerning the amount of basaltic glass available for microbial processing, the principal source of basaltic glass is the upper part of the oceanic crust, of which 500-1000 m consists predominantly of pillow lava. Measurements of a large number of olivine tholeiitic pillows from subglacial sequences in Iceland show an average diameter of $35 \mathrm{~cm}$, with a glass rim thickness of $1 \mathrm{~cm}$ (Friedleifsson et al., 1982). Allowing for $1000 \mathrm{~m}$ of pillow lavas of the above dimensions packed in the closest possible way, and allowing further that about two-thirds of the surface area of the Earth's crust is oceanic, the total volume of basaltic glass (fresh and/or altered) is estimated at $6 \times 10^{6}$ $\mathrm{km}^{3}(1.7 \%$ of the total volume of the pillows that are regarded as spheres) and the surface would cover an area of $3 \times 10^{12} \mathrm{~km}^{2}$. This area is about 4 orders of magnitude larger than the surface of the Earth $\left(3.6 \times 10^{8} \mathrm{~km}^{2}\right)$.

\section{Rate of Microbial Processing of Basaltic Glass}

Concerning the rate of microbial processing of basaltic glass, our experimental studies so far indicate that it may be high (Thorseth et al., in press). In a natural environment such as the oceanic crust, the net effect of microbial activity is probably much slower. The process must have been discontinuous, interrupted by eruptions of new lava that would sterilize the old surface and at the same time bury organicbearing sediments that had accumulated after the previous eruption. In order for the microbial process to proceed, recolonization of the glass surface is required. The encapsulated organic material would provide a substrate for bacterial growth. Nevertheless, the rate of microbial glass dissolution is probably limited by the supply of energy or organic carbon.

The demonstration of cells in 6-m.y.-old basaltic glass in the oceanic crust suggests that large portions of the oceanic crust that contain pillow lava are subjected to microbial weathering of the glass. Temperature and pressure conditions in the upper $1000 \mathrm{~m}$ of the oceanic crust would not prevent bacterial growth. Although the mechanism for energy and carbon supply for microbial growth, the identity of the microbes, and the microbes' affinity for glass surfaces remain to be shown, their presence in the deep oceanic crust shows the existence of a deep subterranean biosphere of large dimensions.

\section{Implications for the Oceanic Crust-Seawater Budget}

Basaltic glass is a major and most easily degradable component of the upper oceanic crust, and its importance for the oceanic crust-seawater budget is well known (e.g., Staudigel and Hart, 1983). In this account we do not attempt any calculation of elemental budget, but would like to emphasize that the occurrence of microbes within the basaltic glass and the way they behave may add to a better understanding of the highly complicated alteration process. We have suggested that microbes may rapidly dissolve basaltic glass, but that the microchannels are subsequently filled with amorphous material. Microprobe analyses of the microbially processed basaltic glass show that all elements vary considerably. The presence of microbes may explain several processes controlling the observed element behavior. Microbes tend to accumulate $\mathrm{Mg}$ or $\mathrm{K}$, whereas $\mathrm{Na}$ is pumped out of the cell (Harder and Dijkhuisen, 1983; Skulachev, 1992). The cells may also 

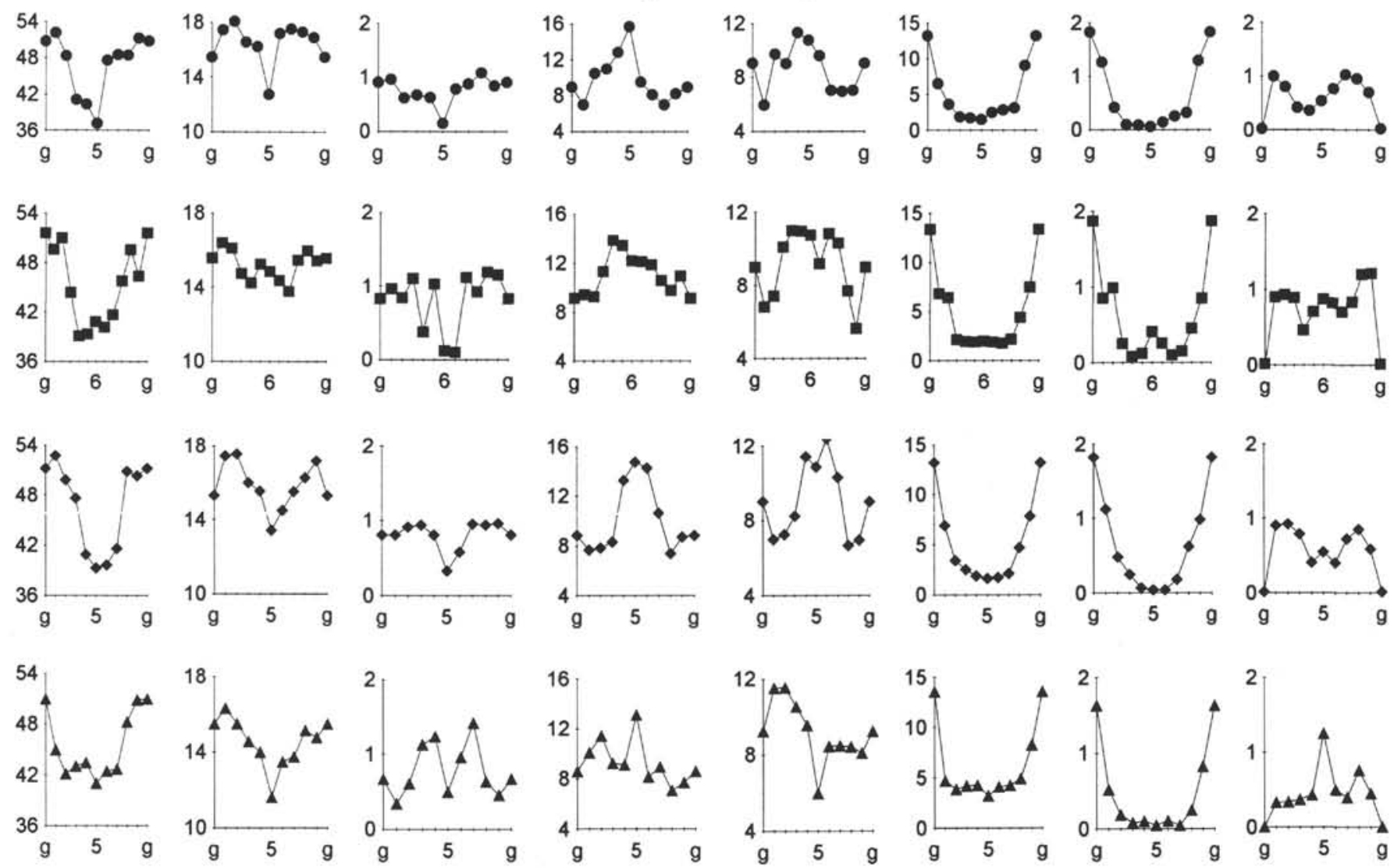

Figure 7. Profiles for $\mathrm{SiO}_{2}, \mathrm{Al}_{2} \mathrm{O}_{3}, \mathrm{TiO}_{2}, \mathrm{FeO}(\mathrm{t}), \mathrm{MgO}, \mathrm{CaO}, \mathrm{Na}_{2} \mathrm{O}$, and $\mathrm{K}_{2} \mathrm{O}$ across the microbially processed area on each side of a joint (shown in Pl. 5) from the four investigated samples. Circles $=$ Sample 148-896A-9R-1, 17-21 cm: squares $=$ Sample 148-896A-11R-1, 73-75 cm; diamonds $=$ Sample $148-896 \mathrm{~A}-$ IIR-I, 1II-I13 cm; and triangles = Sample 148-896A-27R-1, 114-117 cm. The vertical axis of each plot is the weight percent of oxide; the horizontal axis is number of analyses, of which the first and last represent the average fresh glass analysis of each sample and the number identifies the central vein of each analyzed profile.

accumulate $\mathrm{Al}, \mathrm{Si}, \mathrm{Fe}$, and $\mathrm{Ti}$, as well as cause precipitation of various amorphous hydroxides (Ferris et al., 1986; Juniper and Fouquet, 1988; Folk, 1994). Thus, the elements released from the glass may not be lost, but be partially stored where the dissolution took place.

The results presented above strongly suggest that the initial step in, and perhaps a substantial part of, alteration of basaltic glass is a result of microbial activity. The consequences this may have in terms of element loss from the crust to the ocean or vice versa is difficult to estimate, unless the precise amount and composition of secondary minerals of the oceanic crust are known. The extreme accumulation of $\mathrm{K}$, which is most pronounced adjacent to the fresh glass, must, however, have been concentrated by capture from seawater.

\section{SUMMARY}

The main results of this study, focusing on the textures and chemistries of altered basaltic glass from the rim of four pillow basalts at depths from 72 to $237 \mathrm{~m}$ in the volcanic basement, can be summarized as follows:

1. Along cracks in the 1- to 2-cm-thick glass rim of the pillows, there are zones and irregular patches of altered glass that consist of aggregates of (a) a dominant group of small spherical bodies $0.5-1$ $\mu \mathrm{m}$ in diameter (type 1) that define patches up to $50 \mu \mathrm{m}$ thick; (b) larger spherical bodies $2-4$ and $5-10 \mu \mathrm{m}$ in diameter (types 2 and 3 , respectively); and (c) vermicular bodies (type 4) up to $70 \mu \mathrm{m}$ long. The size and shape defined by these bodies are strongly indicative of microbial etching. Further, the presence of DNA, combined with extreme enrichment of $\mathrm{K}$ within these bodies, verifies the existence of viable cells. These observations establish the existence of a volcanic subterranean biosphere.

2. The chemistries of the four groups of differently sized or shaped bodies show large within-group variations. Compared to the host basaltic glass, the general trend is invariable depletion of $\mathrm{CaO}$ and $\mathrm{Na}_{2} \mathrm{O}$; common depletion of $\mathrm{SiO}_{2}$ and $\mathrm{MgO}$; depletion or enrichment of $\mathrm{Al}_{2} \mathrm{O}_{3}, \mathrm{FeO}(\mathrm{t}), \mathrm{TiO}_{2}$, and $\mathrm{P}_{2} \mathrm{O}_{5}$; and invariable enrichment of $\mathrm{K}_{2} \mathrm{O}$, highest adjacent to the fresh glass. Another well-defined chemical feature is that the deepest samples show consistently lower concentrations of $\mathrm{Al}_{2} \mathrm{O}_{3}$ and generally lower concentrations of $\mathrm{FeO}(\mathrm{t})$, $\mathrm{TiO}_{2}$, and $\mathrm{K}_{2} \mathrm{O}$ than the other three.

3 . The above-mentioned textures suggest that microbes dissolve the basaltic glass and live within the micropits and microtubes, where they subsequently accumulate elements in their cell walls or cause the precipitation of elements.

4. This microbial process is important for understanding the chemical budget between the oceanic crust and ocean water. An illustrative example is the extreme enrichment of $\mathrm{K}_{2} \mathrm{O}$ in the microbially processed glass, for which the only possible source is seawater. 


\section{ACKNOWLEDGMENTS}

We thank G. Bratbak, I. Dundas, M. Heldal, R.B. Pedersen, B. Robins, K.P. Skjerlie, and M. Talbot for valuable discussions and constructive criticism of an early draft of the manuscript. We further thank the referees (R. Fisher, P. Michael, and one anonymous), L. Stokking (member of the Editorial Review Board), and C. Fox (member of ODP's Publication Department) for constructive comments. The Norwegian Research Council (NFR) is acknowledged for providing funds for the electron microprobe analyses.

\section{REFERENCES}

Anderson, R.N., Honnorez, J., Becker, K., Adamson, A.C., Alt, J.C., Emmermann, R., Kempton, P.D., Kinoshita, H., Laverne, C., Mottl, M.J., and Newmark, R.L., 1982. DSDP Hole 504B, the first reference section over $1 \mathrm{~km}$ through Layer 2 of the oceanic crust. Nature, 300:589-594.

Berger, G., Schott, J., and Loubet, M., 1987. Fundamental processes controlling the first stage of alteration of a basalt glass by seawater: an experimental study between 200 and $320^{\circ}$ C. Earth Planet. Sci. Lett., 84:431445.

Bernard, F.P., Connan, J., and Magot, M., 1992. Indigenous microorganisms in connate water of many oil fields: a new tool in exploration and production techniques. Annu. Tech. Conf. Soc. Pet. Eng., SPE 24811:467-476.

Brock, T.D., 1985. Life at high temperatures. Science, 29:132-138.

Crovisier, J.L., Honnorez, J., and Eberhart, J.P., 1987. Dissolution of basaltic glass in seawater: mechanism and rate. Geochim. Cosmochim. Acta, 51:2977-2990.

Deming, J.W., and Baross, J.A., 1993. Deep sea smokers: windows to a subsurface biosphere. Geochim. Cosmochim. Acta, 57:3219-3230.

Ehrlich, H.L., 1981. Geomicrobiology: New York (Marcel Dekker).

Ferris, F.G., Beveridge, T.J., and Fyfe, W.S., 1986. Iron-silica crystallite nucleation by bacteria in a geothermal sediment. Nature, 320:609-611.

Folk, R.L., 1994. Nannobacteria: major participants in the precipitation of carbonates and other minerals. In Sasowsky, I.D., and Palmer, M.V. (Eds.), Breakthroughs in Karst Geomicrobiology and Redox Geochemistry. Spec. Publ., 1:17-18.

Friedleifsson, I.B., Furnes, H., and Atkins, F.B., 1982. Subglacial volcanics - on the control of magma chemistry on pillow dimensions. J. Volcanol. Geotherm. Res., 13:103-117.

Furnes, H., and El-Anbaawy, M.I.H., 1980. Chemical changes and authigenic mineral formation during palagonitization of a basanite hyaloclastite, Gran Canaria, Canary Islands. Neues Jahrb. Mineral. Abh. 139:279-302.

Gislason, S.R., and Eugster, H.P., 1987. Meteoric water-basalt interactions, I: a laboratory study. Geochim. Cosmochim. Acta, 51:2827-2840.

Golubic, S., 1973. The relationship between blue-green algae and carbonate deposits. In Carr, N., and Whitton, B.A. (Eds.), The Biology of BlueGreen Algae: Boston (Blackwell), 434-472.

Guy, C., and Schott, J., 1989. Multisite surface reaction versus transport control during the hydrolysis of a complex oxide. Chem. Geol., 78:181-204.

Harder, W., and Dijkhuisen, L., 1983. Physical responses to nutrient limitation. Annu. Rev. Microbiol., 37:1-23.

Hay, R.L., and Iijima, A., 1968a. Nature and origin of palagonite tuffs of the Honolulu Group on Oahu, Hawaii. In Coats, R.R., Hay, R.L., and Anderson, C.A. (Eds.), Studies in Volcanology. Mem.-Geol. Soc. Am., 116:331-376.

1968b. Petrology of palagonite tuffs of the Koko Craters, Oahu, Hawaii. Contrib. Mineral. Petrol., 17:141-154.

Hiebert, F.K., and Bennett, P.C., 1992. Microbial control of silicate weathering in organic rich waters. Science, 258:278-281.

Honnorez, J., 1972. La palagonitisation, l'alteration sous marine du verre volcanique basique de Palagonia (Sicile). Vulkaninstitut Imanuel Friedlander No. 9, Birkhausen Verlag, Basel.

Huber, R., Kurr, M., Jannasch, H.W., and Stetter, K.O., 1989. A novel group of abyssal methanogenic archaebacteria (Methanopyrus) growing at $110^{\circ} \mathrm{C}$. Nature, $342: 833-834$.

Jakobsson, S.P., 1972. On the consolidation and palagonitization of the tephra of the Surtsey volcanic island. Surtsey Prog. Rep., VI:1-8.
1978. Environmental factors controlling the palagonitization of the Surtsey tephra, Iceland. Bull. Geol. Soc. Den., 27:91-105.

Jannasch, H.W., and Mottl, M.J., 1985. Geomicrobiology of deep-sea hydrothermal vents. Science, 229:717-725.

Jannasch, H.W., and Taylor, C.D., 1984. Deep-sea microbiology. Annu. Rev. Microbiol., 38:487-514.

Jones, B., and Goodbody, Q.H., 1982. The geological significance of endolithic algae in glass. Can. J. Earth Sci., 19:671-678.

Juniper, S.K., and Fouquet, Y., 1988. Filamentous iron-silica deposits from modern and ancient hydrothermal sites. Can. Mineral, 26:859-869.

Krumbein, W.E., 1983. Microbial Geochemistry: Oxford (Blackwell Sci.).

Moore, J.G., 1966. Rate of palagonitization of submarine basalt adjacent to Hawaii.Geol. Surv. Prof. Pap. U.S., 550D:163-171.

Noack, Y., 1979. Altération sous-marine des verres volcaniques basiques. Essai sur la palagonitisation [Docteur Spécialité Géologie]. Univ, Louis Pasteur, Strasbourg.

Parkes, P., and Maxwell, J., 1993. Some like it hot (and oily). Nature, 365:694-695.

Paul, A., and Zaman, M.S., 1978. The relative influence of $\mathrm{Al}_{2} \mathrm{O}_{3}$ and $\mathrm{Fe}_{2} \mathrm{O}_{3}$ on the chemical durability of silicate glasses at different $\mathrm{pH}$ values. $J$. Mater. Sci., 13:1499-1502.

Peacock, M.A., 1926. The petrology of Iceland, Part I: the basic tuffs. Trans. R. Soc. Edinburgh, 55:51-76.

Peschek, G.A., 1987. Respiratory electron transport. In Fay, P., and Van Baalen, C. (Eds.), The Cyanobacteria: New York (Elsevier), 119-161.

Porter, K., and Feig, Y.S., 1980. The use of DAPI for identifying and counting aquaric microorganisms. Limnol. Oceanogr., 28:1062-1074.

Rosnes, J.T., Torsvik, T., and Lien, T., 1991. Spore-forming thermophilic sulfate-reducing bacteria isolated from North Sea oil field waters. Appl. Enviroment. Microbiol., 57:2302-2307.

Ross, K.A., and Fisher, R.V., 1986. Biogenic grooving on glass shards. Geology, 14:57I-573.

Shipboard Scientific Party, 1993. Site 896. In Alt, J.C., Kinoshita, H., Stokking, L.B., et al., Proc. ODP, Init. Repts., 148: College Station, TX (Ocean Drilling Program), 123-192.

Skulachev, P., 1992. The law of cell energetics. Eur. J. Biochem., 208:203209.

Staudigel, H., and Hart, S.R., 1983. Alteration of basaltic glass: mechanisms and significance of the oceanic crust-seawater budget. Geochim. Cosmochim. Acta, 47:337-350.

Stetter, K.O., Huber, R., Blöchl, E., Kurr, M., Eden, R.E., Fielder, M., Cash, H., and Vance, I., 1993. Hyperthermophilic archaea are thriving in deep North Sea and Alaskan oil reservoirs. Nature, 365:743-745.

Stokes, K.R., 1971. Further investigations into the nature of the materials chlorophaeite and palagonite. Mineral. Mag., 38:205-214.

Straube, W.L., Deming. J.W., Somerville, C.C., Colwell, R.R., and Baross, J.A., 1990. Particulate DNA in smoker fluids: evidence for existence of microbial populations in hot hydrothermal systems. Appl. Environ. Microbiol., 56:1440-1447.

Thorseth, I.H., Furnes, H., and Heldal. M., 1992a. The importance of microbiological activity in the alteration of natural basaltic glass. Geochim. Cosmochim. Acta, 56:845-850.

Thorseth, I.H., Furnes, H., and Tumyr, O., 1991. A textural and chemical study of Icelandic palagonite of varied composition and its bearing on the mechanism of the glass-palagonite transformation. Geochim. Cosmochim. Acta, 55:731-749.

in press. Textural and chemical effects of bacterial activity on basaltic glass: an experimental approach. Chem. Geol.

Thorseth, I.H., Furnes, H., Tumyr, O., Heldal, M., and Jakobsson, S.P., 1992. Microbiological activity in the alteration of some Icelandic hyaloclastites. In Geirsdottir, A., Norddal, A., and Helgadottir, H. (Eds.), 20th Nordic Geol. Winter Meeting. Reykjavik (Univ. Iceland), 170. (Abstract)

Date of initial receipt: 25 July 1994

Date of acceptance: 15 November 1994

Ms 148SR-119 

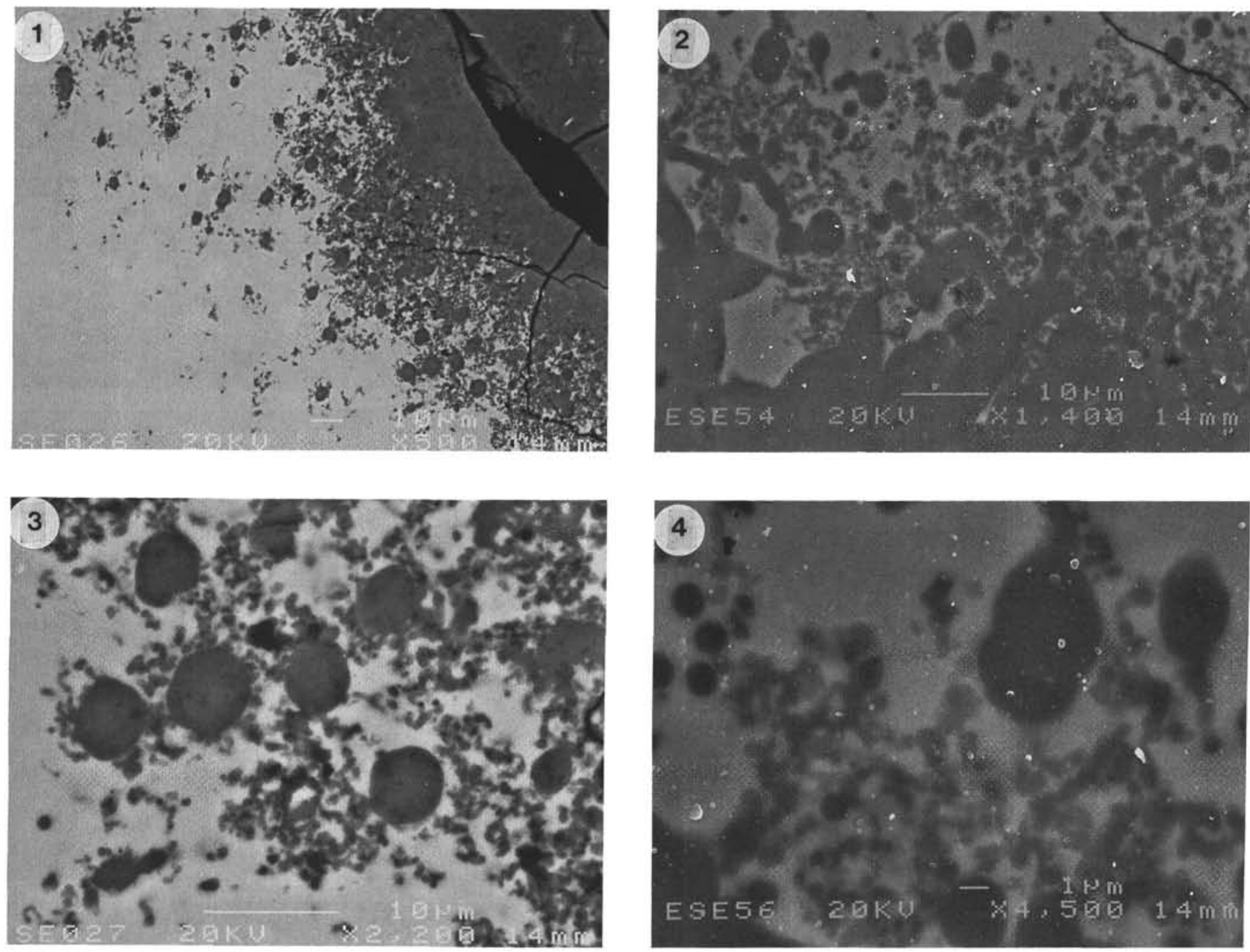

Plate 1. BSE-SEM photomicrographs of an altered glass rim of pillow lava. All samples contain fractures, along which the degree of alteration is highly variable, from very incipient to advanced stages of alteration. The development of alteration patches on only one side of the fractures in all samples is particularly well exhibited in Figures 2 and 4 . The sausage-like alteration pattern is shown in Figure 3. The fresh basaltic glass is light gray, the altered parts are dark gray, and crystals (all plagioclase) have an intermediate gray color. 1. Sample 148-896A-9R-1, 17-21 cm. 2. Sample 148-896A-11R-1, 73-75 cm. 3. Sample 148896A-11R-1, 111-113 cm. 4. Sample 148-896A-27R-1, II4-117 cm. 

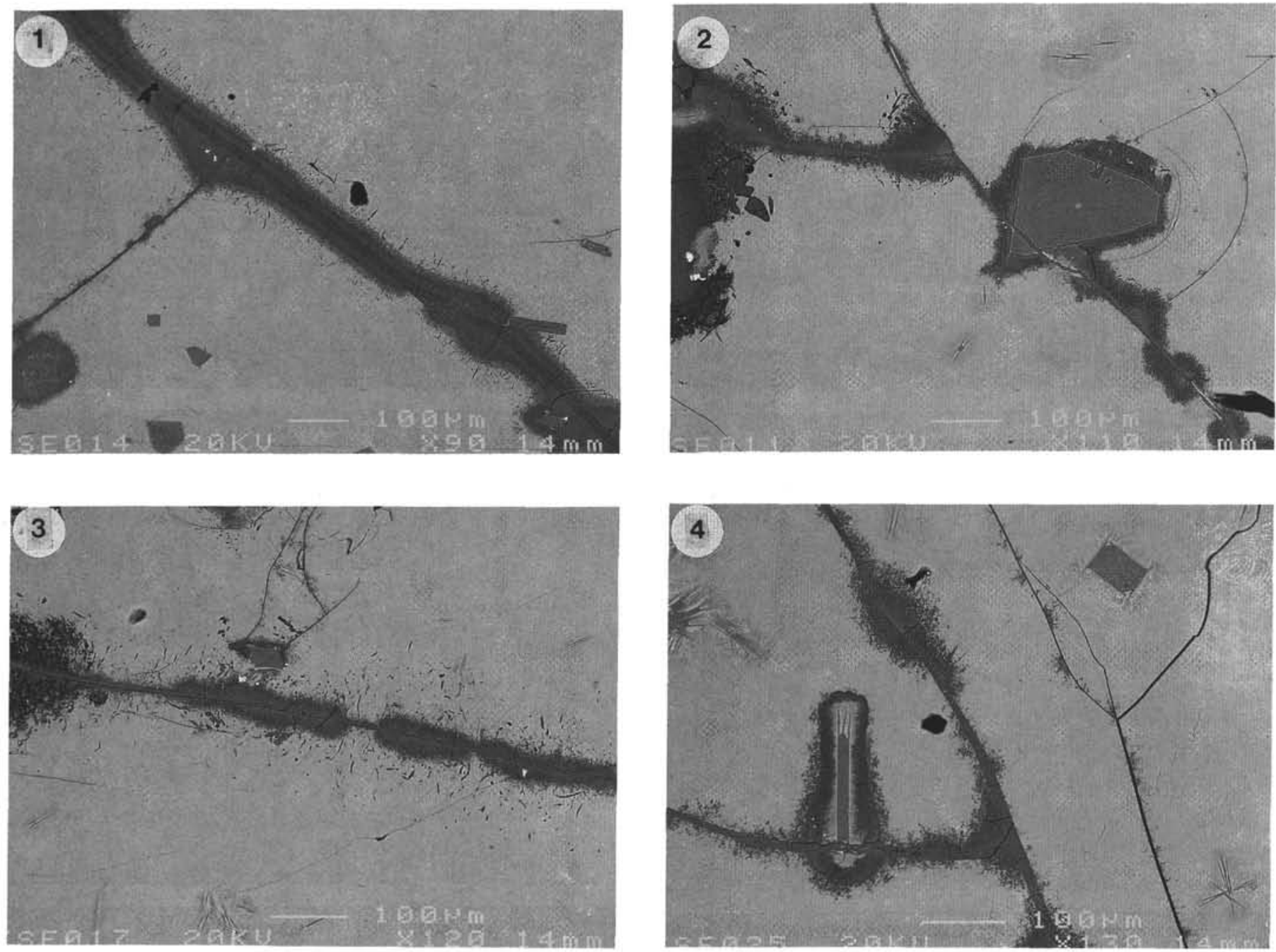

Plate 2. BSE-SEM photomicrographs of Sample 148-896A-27R-1, 114-117 cm, showing the general appearance (Figs. 1,2) and details (Figs. 3, 4) of the three different-sized spherical alteration objects (types 1,2, and 3). Figure 3 shows types 1 ( $0.5-1 \mu \mathrm{m}$ in diameter) and $3(5-10 \mu \mathrm{m}$ in diameter), whereas Figure 4 shows types 1 and $2(2-3 \mu \mathrm{m}$ in diameter). Fresh glass is light gray, whereas altered glass is dark gray. 

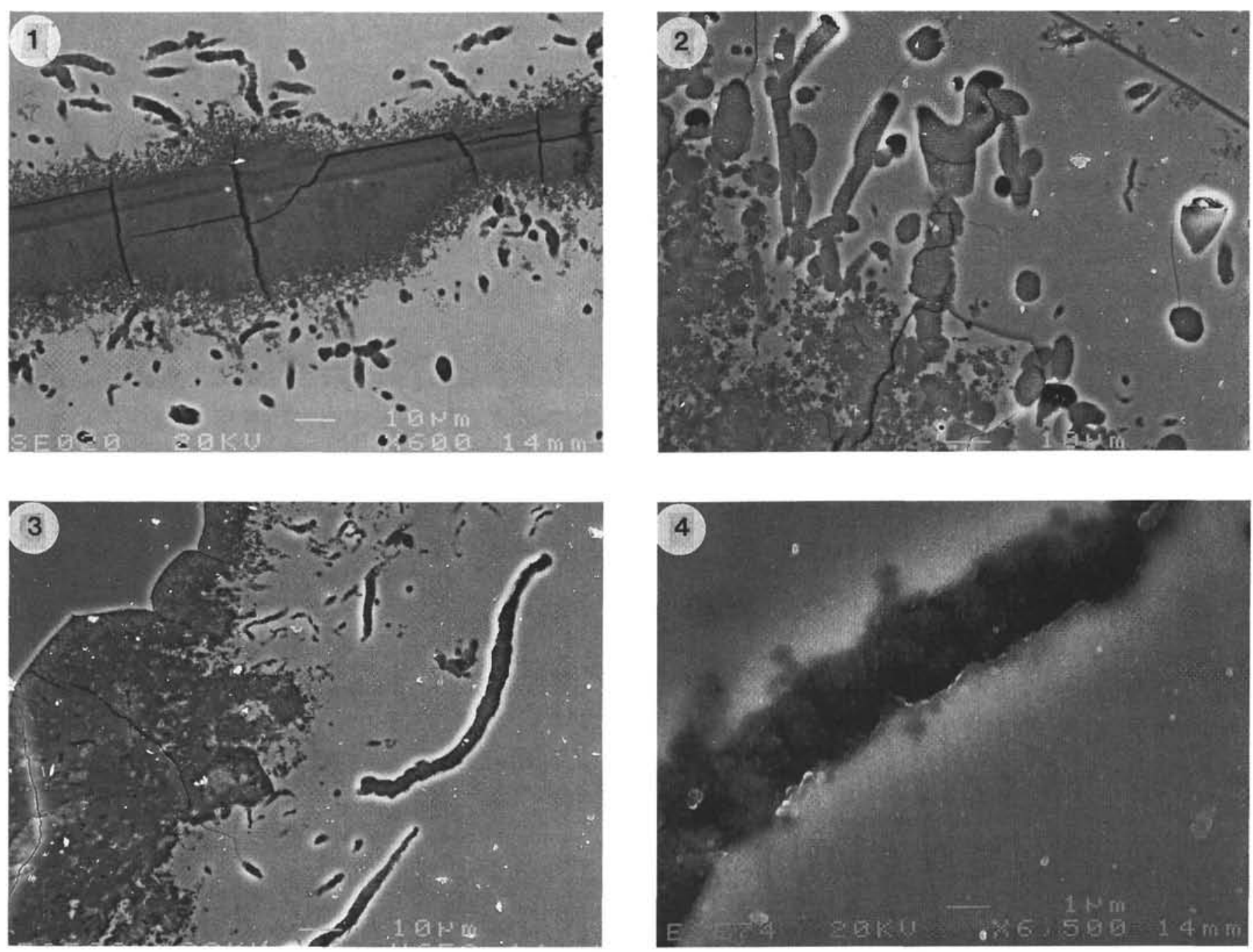

Plate 3. BSE-SEM photomicrographs showing the general appearance (Fig. 1. Sample 148-896A-1IR-1, 111-113 cm) and details (Figs. 2-4, Sample 148$896 \mathrm{~A}-1 \mathrm{IR}-\mathrm{I}, 73-75 \mathrm{~cm}$ ) of the vermicular-shaped alteration objects (type 4). Fresh glass is light gray, whereas altered glass is dark gray. 

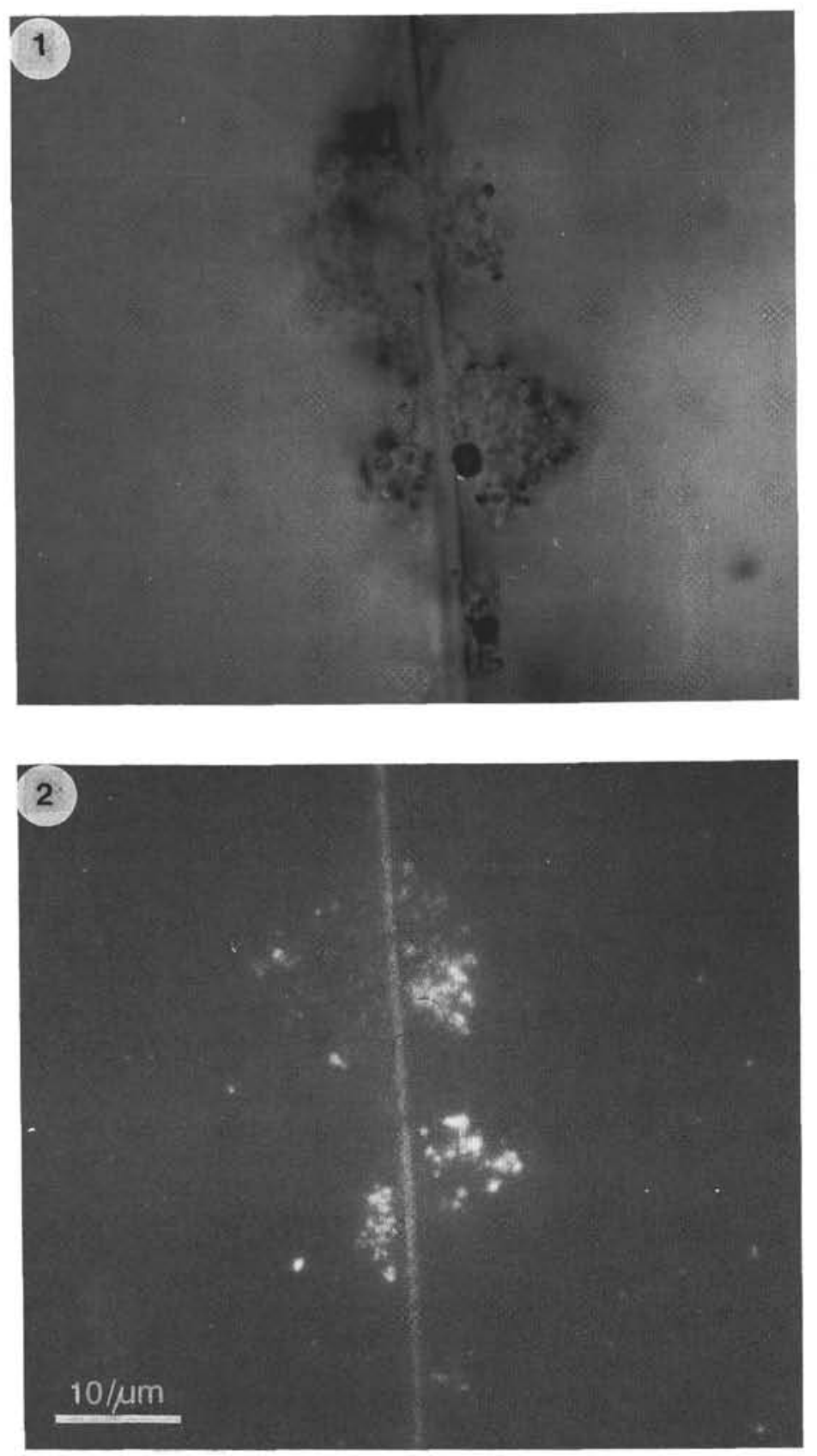

Plate 4. Microphotographs of bacteria in basaltic glass (Sample 148-896A-IIR-1, 73-73 cm). Sample examined in a Nikon Microphot microscope using (1) light transmission and (2) epifluorescence with an excitation light at $365 \mathrm{~nm}$ and emission at $420 \mathrm{~nm}$. 

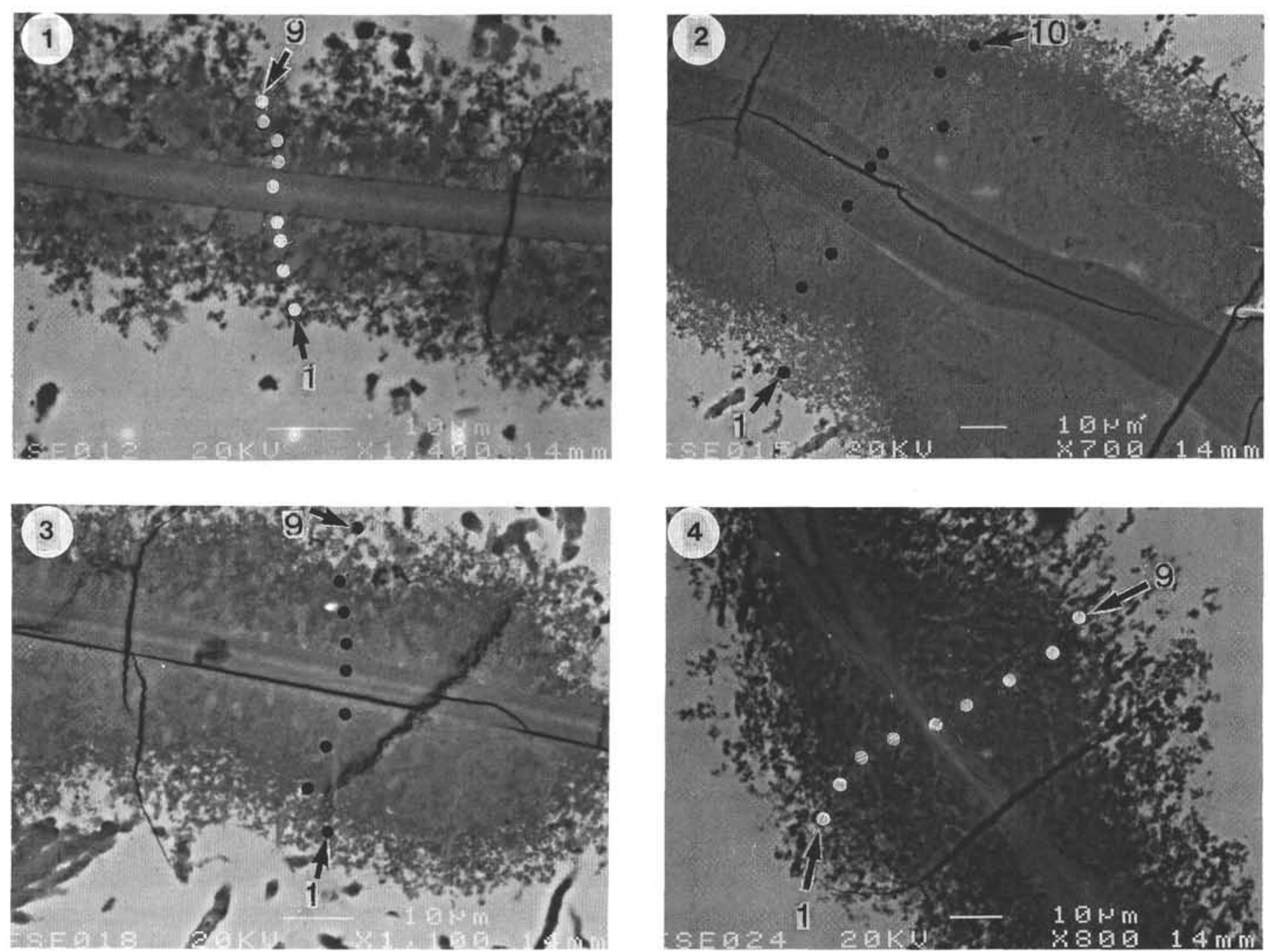

Plate 5. BSE-SEM photomicrographs showing the traverses of the microprobe analyses shown in Table 3 and illustrated in Figure 7. 1. Sample 148-896A-9R-1, 17-21 cm. 2. Sample 148-896A-11R-1, 73-75 cm. 3. Sample 148-896A-11R-1, 111-113 cm. 4. Sample 148-896A-27R-1, 114-117 cm. The number of each analyzed spot corresponds to that in Table 3 . At the alteration front, the bodies typically have the morphology and size of type 1 . 